\title{
Recoverable (R)- and (S)-Binap-Ag(I) Complexes for the Enantioselective 1,3-Dipolar Cycloaddition Reaction of Azomethine Ylides ${ }^{\S}$
}

\author{
Carmen Nájera,* M. de Gracia Retamosa and José \\ M. Sansano* \\ Departamento de Química Orgánica and Instituto de \\ Síntesis Orgánica (ISO). Facultad de Ciencias, \\ Universidad de Alicante \\ 03080-Alicante (Spain) \\ Fax: (+34) 965903549 \\ E-mail:cnajera@ua.es;jmsansano@ua.es
}

General Remarks: All reactions were carried out in the absence of light. Anhydrous solvents were freshly distilled under an argon atmosphere. Aldehydes were also distilled prior to use for the elaboration of the imino esters. Melting points were determined with a Reichert Thermovar hot plate apparatus and are uncorrected. Only the structurally most important peaks of the IR spectra (recorded on a Nicolet 510 P-FT) are listed. ${ }^{1} \mathrm{H}$ NMR (300 MHz) and ${ }^{13} \mathrm{C}$ NMR (75 MHz) spectra were obtained on a Bruker AC-300 using $\mathrm{CDCl}_{3}$ as solvent and TMS as internal standard, unless otherwise stated. Optical rotations were measured on a Perkin Elmer 341 polarimeter. HPLC analyses were performed on a JASCO 2000-series equipped with a chiral column (detailed for each compound in the main text), using mixtures of n-hexane/isopropyl alcohol as mobile phase, at $25{ }^{\circ} \mathrm{C}$. Low-resolution electron impact (EI) mass spectra were obtained at $70 \mathrm{eV}$ on a Shimadzu QP-5000 and high-resolution mass spectra were obtained on a Finnigan VG Platform. HRMS (EI) were recorded on a Finnigan MAT 95S. Microanalyses were performed on a Perkin Elmer 2400 and a Carlo Erba EA1108. Analytical TLC was performed on Schleicher \& Schuell F1400/LS silica gel plates and the spots were visualised under UV light ( $=254 \mathrm{~nm}$ ). For flash chromatography we employed Merck silica gel 60 (0.040-0.063 mm).

\footnotetext{
${ }^{\S}$ Dedicated to Professors Juan Forniés and José Gimeno on the occasion of their $60^{\text {th }}$ birthday.
} 
General procedure: A solution of the imino ester 4 (1 mmol) and maleimide (1 mmol) in toluene $(5 \mathrm{~mL})$ was added to a suspension of complex $7(0.05 \mathrm{mmol}, 41 \mathrm{mg})$ in toluene $(5 \mathrm{~mL})$ or to an in situ generated complex formed by mixing $(R)$ - or $(S)$-binap (0.05 mmol, $31 \mathrm{mg})$ and $\mathrm{AgClO}_{4}(0.05 \mathrm{mmol}, 10 \mathrm{mg})$ in toluene $(5 \mathrm{~mL})$. To the resulting suspension triethylamine $(0.05 \mathrm{mmol}, 7 \mu \mathrm{L})$ was added and the mixture stirred at room temperature and in the absence of the light for $16 \mathrm{~h}$. When DBU was used as base (Table 3, entries 6 and 9) the reaction was run at $0{ }^{\circ} \mathrm{C}$ or $-20{ }^{\circ} \mathrm{C}$ for $1 \mathrm{~d}$. The precipitate was filtered and the complex was recovered. ${ }^{25}$ The organic filtrate was directly evaporated and the residue was purified by recrystallization or by flash chromatography yielding pure products $6,9,10$, or 11 .

The solid was washed with warm toluene twice and then dissolved in DCM in order to transfer the catalytic complex $(S)-7$ into the flask. After evaporation of DCM the resulting solid was ready to catalyze a new batch.

Analytical and physical data of new compounds are the following:

\section{Methyl (1S,3R,3aS,6aR)-5-methyl-3-phenyl-4,6-dioxooctahydropyrrolo[3,4- c]pyrrole-1-carboxylate (6a). ${ }^{1}$}

Methyl (1S,3R,3aS,6aR)-5-methyl-3-(2-naphthyl)-4,6-dioxooctahydropyrrolo[3,4c]pyrrole-1-carboxylate (6b):

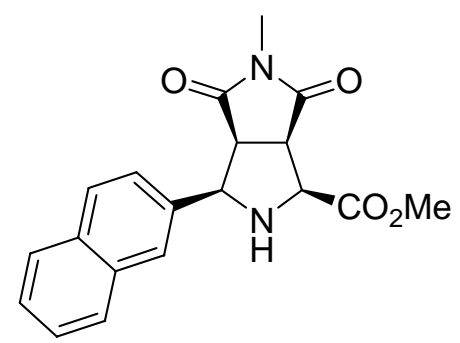

Colorless prisms, mp $=>195{ }^{\circ} \mathrm{C}$ (subl) $\left(\mathrm{CH}_{2} \mathrm{Cl}_{2} / \mathrm{Hex}\right) .[\alpha]_{\mathrm{D}}{ }^{20}=+109.4\left(c 1, \mathrm{CHCl}_{3}\right) .99 \%$ ee from HPLC (Chiralpak AS, $1 \mathrm{~mL} / \mathrm{min}, n$-hexane $/ i-\mathrm{PrOH}: 20 / 80, \lambda 225 \mathrm{~nm}$ ), $\mathrm{t}_{\mathrm{Rmaj}}=$ $8.6 \mathrm{~min}, \mathrm{t}_{\mathrm{Rmin}}=26.7 \mathrm{~min} . R_{\mathrm{f}}: 0.42$ ( $n$-hexane/ethyl acetate: 1/5). IR (KBr) v. 1758, 1729, 1697, $3339 \mathrm{~cm}^{-1} .{ }^{1} \mathrm{H}$ RMN $\delta_{\mathrm{H}}: 2.46(\mathrm{dd}, J=4.8,4.8 \mathrm{~Hz}, 1 \mathrm{H}, \mathrm{NH}), 2.83\left(\mathrm{~s}, 3 \mathrm{H}, \mathrm{NCH}_{3}\right.$ ), 3.46 (dd, $J=7.9,8.2 \mathrm{~Hz}, 1 \mathrm{H}, \mathrm{CHCHAr}$ ), 3.55 (dd, $J=7.3,7.0 \mathrm{~Hz}, 1 \mathrm{H}, \mathrm{CHCHCO}_{2} \mathrm{CH}_{3}$ ), 3.89 (s, $\left.3 \mathrm{H}, \mathrm{CO}_{2} \mathrm{CH}_{3}\right), 4.02$ (dd, $\left.J=6.6,5.3 \mathrm{~Hz}, 1 \mathrm{H}, \mathrm{CHCO}_{2} \mathrm{CH}_{3}\right), 4.56$ (dd, $J=8.2$, $5.3 \mathrm{~Hz}, 1 \mathrm{H}, \mathrm{CHAr}), 7.39-7.47$ (m, 3H, ArH), 7.78-7.82 (m, 4H, ArH). ${ }^{13} \mathrm{C} \mathrm{RMN} \delta_{\mathrm{C}}$ : $25.0\left(\mathrm{NCH}_{3}\right), 48.1,49.2$, $52.3\left(2 \mathrm{CHCON}\right.$ and $\left.\mathrm{CO}_{2} \mathrm{CH}_{3}\right), 61.6\left(\mathrm{CHCO}_{2} \mathrm{Me}\right), 63.9$ (2Naft-CH),125.2, 125.4, 126.0, 126.2, 127.8, 127.9, 127.9 (ArCH), 133.2, 134.2 (ArC), 170.1, 174.6, 175.9 ( $\mathrm{CO}_{2} \mathrm{Me}$ and CON). MS (EI) $\mathrm{m} / z$ (\%): $338\left(\mathrm{M}^{+}, 21.29 \%\right), 228$ (10), 227(64), 196 (34), 192 (13), 168 (34), 167 (100), 166 (12), 165 (16), 140 (16) 139 (14), 97 (23). HRMS calcd. for $\mathrm{C}_{19} \mathrm{H}_{18} \mathrm{~N}_{2} \mathrm{O}_{4}$ : 338.1267; found: 338.1263 .

\footnotetext{
${ }^{1}$ Dogan, O.; Koyuncu, H.; Garner, P.; Bulut, A.; Youngs, W. J.; Panzner, M. Org. Lett. 2006, 8, 4687.
} 
Methyl (1S,3R,3aS,6aR)-3-(2-chlorophenyl)-5-methyl-4,6dioxooctahydropyrrolo[3,4-c]pyrrole-1-carboxylate (6c):<smiles>CC(=O)C1N[C@H](c2ccccc2Cl)[C@@H]2C(=O)N(C)C(=O)[C@H]12</smiles>

Colorless prisms, $\mathrm{mp}=>180{ }^{\circ} \mathrm{C}$ (subl) $\left(\mathrm{CH}_{2} \mathrm{Cl}_{2} / \mathrm{Hex}\right) .[\alpha]_{\mathrm{D}}{ }^{20}=+27.1\left(c 1, \mathrm{CHCl}_{3}\right) .85 \%$ $e e$ from HPLC (Chiralcel ODH, $1 \mathrm{~mL} / \mathrm{min}, n$-hexane $/ i$-PrOH: 70/30, $\lambda 215 \mathrm{~nm}$ ), $\mathrm{t}_{\mathrm{Rmin}}=$ $24.9 \mathrm{~min}, \mathrm{t}_{\mathrm{Rmaj}}=34.2 \mathrm{~min} . R_{\mathrm{f}}$ : 0.46 ( $n$-hexane/ethyl acetate: 1/5). IR (KBr) v. 1744, 1705, $3346 \mathrm{~cm}^{-1} .{ }^{1} \mathrm{H}$ RMN $\delta_{\mathrm{H}}: 2.32$ (dd, $J=4.4,3.8 \mathrm{~Hz}, 1 \mathrm{H}, \mathrm{NH}$ ), 2.82 (s, 3H, $\mathrm{NCH}_{3}$ ), 3.58 (dd, $J=7.5,7.1 \mathrm{~Hz}, 1 \mathrm{H}, \mathrm{CHCHAr}$ ), 3.73 (dd, $J=8.0,8.0 \mathrm{~Hz}, 1 \mathrm{H}, \mathrm{CHCHCO}_{2} \mathrm{CH}_{3}$ ), 3.88 (s, $3 \mathrm{H}, \mathrm{CO}_{2} \mathrm{CH}_{3}$ ), 4.08 (dd, $J=6.9,4.7 \mathrm{~Hz}, 1 \mathrm{H}, \mathrm{CHCO}_{2} \mathrm{CH}_{3}$ ), 4.76 (dd, $J=8.4$, 4.9, Hz, 1H, CHAr), 7.22-7.28 (m, 2H, ArH), 7.40-7.42 (m, 1H, ArH), 7.51-7.53 (m, $1 \mathrm{H}, \mathrm{ArH}) .{ }^{13} \mathrm{C} \mathrm{RMN} \delta_{\mathrm{C}}: 24.9\left(\mathrm{NCH}_{3}\right), 46.5,47.6(2 \mathrm{CHCON}), 52.3\left(\mathrm{CO}_{2} \mathrm{CH}_{3}\right), 60.3$ $\left(\mathrm{CHCO}_{2} \mathrm{Me}\right), 61.2$ (o-ClPh-CH), 126.9, 127.2, 129.1, $129.2(\mathrm{ArCH}), 133.2,134.8$ (ArC), 170.1, 174.3, 175.9 ( $\mathrm{CO}_{2} \mathrm{Me}$ and $\left.\mathrm{CON}\right)$. MS (EI) $m / z(\%): 322\left(\mathrm{M}^{+}, 6.33 \%\right), 265$ (33), 264 (16), 263 (100), 213 (25), 211 (76), 180 (26), 179 (16), 178 (48), 153 (25), 152 (12), 151 (74), 143 (17), 115 (19), 114 (12). HRMS calcd. for $\mathrm{C}_{15} \mathrm{H}_{15} \mathrm{ClN}_{2} \mathrm{O}_{4}$ : 322.0720; found: 322.0720 .

Methyl (1S,3R,3aS,6aR)-5-methyl-3-(4-methylphenyl)-4,6dioxooctahydropyrrolo[3,4-c]pyrrole-1-carboxylate (6d):<smiles>CC(=O)[C@@H]1N[C@H](c2ccc(C)cc2)[C@H]2C(=O)N(C)C(=O)[C@@H]21</smiles>

Colorless prisms, $\mathrm{mp}=>155{ }^{\circ} \mathrm{C}(\mathrm{dec})\left(\mathrm{CH}_{2} \mathrm{Cl}_{2} / \mathrm{Hex}\right) .[\alpha]_{\mathrm{D}}{ }^{20}=+89\left(c 0.4, \mathrm{CHCl}_{3}\right) .99 \%$ ee from HPLC (Chiralpak AS, $1 \mathrm{~mL} / \mathrm{min}, n$-hexane $/ i-\mathrm{PrOH}: 20 / 80, \lambda 215 \mathrm{~nm}$ ), $\mathrm{t}_{\mathrm{Rmaj}}=$ $7.3 \mathrm{~min}, \mathrm{t}_{\mathrm{Rmin}}=16.5 \mathrm{~min} . R_{\mathrm{f}}: 0.38$ ( $n$-hexane/ethyl acetate: 1/5). IR (KBr) v. 1739, 1694, $3338 \mathrm{~cm}^{-1} .{ }^{1} \mathrm{H}$ RMN $\delta_{\mathrm{H}}: 2.34$ (s, 3H, $\mathrm{PhCH}_{3}$ ), (dd, $\left.J=5.3,5.1 \mathrm{~Hz}, 1 \mathrm{H}, \mathrm{NH}\right), 2.87$ (s, 3H, $\mathrm{NCH}_{3}$ ), 3.40 (dd, $J=8.2,8.0 \mathrm{~Hz}, 1 \mathrm{H}, \mathrm{CHCHAr}$ ), 3.55 (dd, $J=7.3,7.1 \mathrm{~Hz}, 1 \mathrm{H}$, $\mathrm{CHCHCO}_{2} \mathrm{CH}_{3}$ ), 3.87 (s, $3 \mathrm{H}, \mathrm{CO}_{2} \mathrm{CH}_{3}$ ), 4.03 (dd, $J=6.6,5.7 \mathrm{~Hz}, 1 \mathrm{H}, \mathrm{CHCO}_{2} \mathrm{CH}_{3}$ ), 4.45 (dd, $J=8.4,5.7 \mathrm{~Hz}, 1 \mathrm{H}, \mathrm{CHAr}), 7.15$ (d, $J=7.9 \mathrm{~Hz} 2 \mathrm{H}, \mathrm{ArH}), 7.21$ (d, $J=8.0 \mathrm{~Hz}$ $2 \mathrm{H}, \mathrm{ArH}) .{ }^{13} \mathrm{C} \mathrm{RMN} \delta_{\mathrm{C}}: 21.2\left(\mathrm{PhCH}_{3}\right), 24.9\left(\mathrm{NCH}_{3}\right), 48.2,49.5(2 \mathrm{CHCON}), 52.3$ $\left(\mathrm{CO}_{2} \mathrm{CH}_{3}\right), 61.6\left(\mathrm{CHCO}_{2} \mathrm{Me}\right), 63.9$ ( $\left.p-\mathrm{MePh}-\mathrm{CH}\right), 126.9,129.1(\mathrm{ArCH}), 133.5,137.9$ (ArC), 170.1, 174.8, $176.0\left(\mathrm{CO}_{2} \mathrm{Me}\right.$ and CON). MS (EI) m/z (\%): 302 (M , 10.52\%), 243 (60), 241 (12), 192 (12), 191 (95), 160 (20), 159 (19), 158 (36), 156 (14), 132 (16), 131 (100), 130 (15). HRMS calcd. for $\mathrm{C}_{16} \mathrm{H}_{18} \mathrm{~N}_{2} \mathrm{O}_{4}$ : 302.1267; found: 302.1270 .

Methyl (1S,3R,3aS,6aR)-3-(4-methoxyphenyl)-5-methyl-4,6dioxooctahydropyrrolo[3,4-c]pyrrole-1-carboxylate (6e): 
<smiles>COc1ccc([C@@H]2N[C@H](C(C)=O)[C@H]3C(=O)N(C)C(=O)[C@@H]32)cc1</smiles>

Colorless prisms, mp $=>205^{\circ} \mathrm{C}\left(\mathrm{CH}_{2} \mathrm{Cl}_{2} / \mathrm{Hex}\right)$ (subl). $[\alpha]_{\mathrm{D}}{ }^{20}=+114\left(c \mathrm{c}\right.$.5, $\left.\mathrm{CHCl}_{3}\right) .99 \%$ $e e$ from HPLC (Chiralcel ODH, $1 \mathrm{~mL} / \mathrm{min}, n$-hexane $/ i$-PrOH: 70/30, $\lambda 220 \mathrm{~nm}$ ), $\mathrm{t}_{\mathrm{Rmaj}}=$ $43.2 \mathrm{~min}, \mathrm{t}_{\mathrm{Rmin}}=50.1 \mathrm{~min} . R_{\mathrm{f}}: 0.29$ (n-hexane/ethyl acetate: 1/5). IR $(\mathrm{KBr})$ v. 1748, 1701, $3342 \mathrm{~cm}^{-1} .{ }^{1} \mathrm{H}$ RMN $\delta_{\mathrm{H}}: 2.37$ (dd, $\left.J=5.0,4.8 \mathrm{~Hz}, 1 \mathrm{H}, \mathrm{NH}\right), 2.89$ (s, 3H, $\mathrm{NCH}_{3}$ ), 3.39 (dd, $J=8.1,7.9 \mathrm{~Hz}, 1 \mathrm{H}, \mathrm{CHCHAr}$ ), 3.55 (dd, $J=7.3,7.0 \mathrm{~Hz}, 1 \mathrm{H}, \mathrm{CHCHCO}_{2} \mathrm{CH}_{3}$ ), $3.803 .88\left(2 \mathrm{~s}, 6 \mathrm{H}, \mathrm{OCH}_{3}\right.$ and $\left.\mathrm{CO}_{2} \mathrm{CH}_{3}\right), 4.04$ (dd, $\left.J=5.6,6.2 \mathrm{~Hz}, 1 \mathrm{H}, \mathrm{CHCO}_{2} \mathrm{CH}_{3}\right), 4.46$ (dd, $J=5.3,8.4 \mathrm{~Hz}, 1 \mathrm{H}, \mathrm{CHAr}$ ), 6.87 (d, $J=8.6 \mathrm{~Hz} 2 \mathrm{H}, \mathrm{ArH}$ ), 7.25 (d, $J=8.6 \mathrm{~Hz} 2 \mathrm{H}$, $\mathrm{ArH}) .{ }^{13} \mathrm{C} \mathrm{RMN} \delta_{\mathrm{C}}: 25.0\left(\mathrm{NCH}_{3}\right), 48.2,49.4(2 \mathrm{CHCON}), 52.3,55.1\left(\mathrm{CO}_{2} \mathrm{CH}_{3}\right.$ and $\left.\mathrm{OCH}_{3}\right), 61.6\left(\mathrm{CHCO}_{2} \mathrm{Me}\right), 63.6(p-\mathrm{MeOPh}-\mathrm{CH}), 113.8,128.1(\mathrm{ArCH}), 128.5,159.4$ (ArC), 170.2, 174.9, 176.0 ( $\mathrm{CO}_{2} \mathrm{Me}$ and CON). MS (EI) $\mathrm{m} / z(\%): 318\left(\mathrm{M}^{+}, 12.27 \%\right)$, 259 (27), 208 (12), 207 (92), 176 (12), 175 (23), 174 (18) 172 (16), 148 (16), 147 (100), 132 (14), 120 (10), 87 (10), HRMS calcd. for $\mathrm{C}_{16} \mathrm{H}_{18} \mathrm{~N}_{2} \mathrm{O}_{5}$ : 318,1216; found: 318.1214.

Methyl (1S,3R,3aS,6aR)-3-(4-chlorophenyl)-5-methyl-4,6dioxooctahydropyrrolo[3,4-c]pyrrole-1-carboxylate (6f):

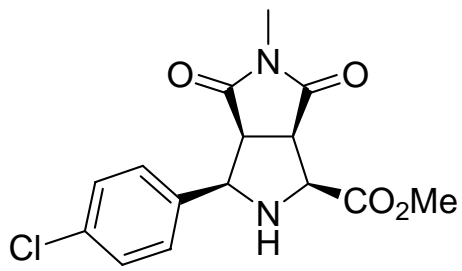

Colorless prisms, $\mathrm{mp}=>215^{\circ} \mathrm{C}(\mathrm{subl})\left(\mathrm{CH}_{2} \mathrm{Cl}_{2} / \mathrm{Hex}\right) \cdot[\alpha]_{\mathrm{D}}{ }^{20}=-28\left(c 0.5, \mathrm{CHCl}_{3}\right) .99 \%$ $e e$ from HPLC (Chiralcel ODH, $1 \mathrm{~mL} / \mathrm{min}, n$-hexane/i-PrOH: 80/20, $\lambda 215 \mathrm{~nm}$ ), $\mathrm{t}_{\text {Rmaj }}$ $=47.6 \mathrm{~min}, \mathrm{t}_{\mathrm{Rmin}}=54.6 \mathrm{~min} . R_{\mathrm{f}}: 0.33$ ( $n$-hexane/ethyl acetate: $\left.1 / 5\right) . \mathrm{IR}(\mathrm{KBr}) v .1744$, 1693, 1682, $3356 \mathrm{~cm}^{-1} .{ }^{1} \mathrm{H}$ RMN $\delta_{\mathrm{H}}: 2.39$ (dd, $\left.J=3.8,4.4 \mathrm{~Hz}, 1 \mathrm{H}, \mathrm{NH}\right), 2.88$ (s, 3H, $\mathrm{NCH}_{3}$ ), 3.39 (dd, $J=8.2,8.0 \mathrm{~Hz}, 1 \mathrm{H}, \mathrm{CHCHAr}$ ), 3.56 (dd, $J=7.3,7.1 \mathrm{~Hz}, 1 \mathrm{H}$, $\mathrm{CHCHCO}_{2} \mathrm{CH}_{3}$ ), 3.87 (s, $3 \mathrm{H}, \mathrm{CO}_{2} \mathrm{CH}_{3}$ ), 4.04 (dd, $J=6.8,4.9 \mathrm{~Hz}, 1 \mathrm{H}, \mathrm{CHCO}_{2} \mathrm{CH}_{3}$ ), 4.46 (dd, $J=8.4,4.9 \mathrm{~Hz}, 1 \mathrm{H}, \mathrm{CHAr}), 7.27-7.33(\mathrm{~m}, 4 \mathrm{H}, \mathrm{ArH}) .{ }^{13} \mathrm{C} \mathrm{RMN} \delta_{\mathrm{C}}: 25.0$ $\left(\mathrm{NCH}_{3}\right)$, 47.9, $49.2(2 \mathrm{CHCON}), 52.3\left(\mathrm{CO}_{2} \mathrm{CH}_{3}\right), 61.5\left(\mathrm{CHCO}_{2} \mathrm{Me}\right), 63.2(p-\mathrm{ClPh}-\mathrm{CH})$, 128.4, 128.6 (ArCH), 133.9, 135.2 (ArC), 169.9, 174.5, $175.7\left(\mathrm{CO}_{2} \mathrm{Me}\right.$ and $\left.\mathrm{CON}\right) . \mathrm{MS}$ (EI) $m / z$ (\%): 322 (M+1 9.56\%), 265 (30), 264 (15), 263 (92), 261 (11), 213 (32), 212 (11), 211 (95), 181 (10), 180 (28), 179 (29), 178 (48), 176 (10), 153 (33), 152 (15), 151 (100), 143 (24), 115 (19). HRMS calcd. for $\mathrm{C}_{15} \mathrm{H}_{15} \mathrm{ClN}_{2} \mathrm{O}_{4}$ : 322.0720; found: 322.0719.

Methyl (1S,3R,3aS,6aR)-5-methyl-4,6-dioxo-3-(2-thienyl)octahydropyrrolo[3,4c]pyrrole-1-carboxylate (6g):<smiles>CC(=O)[C@H]1N[C@H](c2cccs2)[C@H]2C(=O)N(C)C(=O)[C@H]12</smiles>

Colorless prisms, mp $=>196{ }^{\circ} \mathrm{C}$ (subl) $\left(\mathrm{CH}_{2} \mathrm{Cl}_{2} / \mathrm{Hex}\right) .[\alpha]_{\mathrm{D}}{ }^{20}=+42\left(c 0.6, \mathrm{CHCl}_{3}\right) .92 \%$ $e e$ from HPLC (Chiralpak AD, $1 \mathrm{~mL} / \mathrm{min}, n$-hexane $/ i$-PrOH: 70/30, $\lambda 215 \mathrm{~nm}$ ), $\mathrm{t}_{\mathrm{Rmin}}=$ 
$10.8 \mathrm{~min}, \mathrm{t}_{\mathrm{Rmaj}}=14.6 \min . R_{\mathrm{f}}: 0.33$ (n-hexane/ethyl acetate: $\left.1 / 5\right) . \mathrm{IR}(\mathrm{KBr}) v .1738$, 1701, $3344 \mathrm{~cm}^{-1} .{ }^{1} \mathrm{H}$ RMN $\delta_{\mathrm{H}}: 2.63$ (dd, $\left.J=5.1,4.8 \mathrm{~Hz}, 1 \mathrm{H}, \mathrm{NH}\right), 2.90$ (s, 3H, $\mathrm{NCH}_{3}$ ), 3.42 (dd, $J=8.4,8.0 \mathrm{~Hz}, 1 \mathrm{H}, \mathrm{CHCHAr}$ ), 3.56 (dd, $J=6.7,5.5 \mathrm{~Hz}, 1 \mathrm{H}, \mathrm{CHCHCO}_{2} \mathrm{CH}_{3}$ ), 3.87 (s, $3 \mathrm{H}, \mathrm{CO}_{2} \mathrm{CH}_{3}$ ), 4.04 (dd, $J=6.6,5.1 \mathrm{~Hz}, 1 \mathrm{H}, \mathrm{CHCO}_{2} \mathrm{CH}_{3}$ ), 4.82 (dd, $J=$ 8.6, $5.5 \mathrm{~Hz}, 1 \mathrm{H}, \mathrm{CHAr}$ ), 7.01 (dd, $J=5.1,3.6 \mathrm{~Hz}, 1 \mathrm{H}, \mathrm{SCHCHCH}$ ), 7.08 (ddd, $J=3.6,1.1$, $0.8 \mathrm{~Hz}, 1 \mathrm{H}$, SCHCHCH), 7.26 (dd, $J=5.0,1.2 \mathrm{~Hz}, 1 \mathrm{H}$, SCHCHCH). ${ }^{13} \mathrm{C}$ RMN $\delta_{\mathrm{C}}: 25.1$ $\left(\mathrm{NCH}_{3}\right)$, 48.1, $49.6(2 \mathrm{CHCON}), 52.3\left(\mathrm{CO}_{2} \mathrm{CH}_{3}\right), 59.6\left(\mathrm{CHCO}_{2} \mathrm{Me}\right), 61.3$ (2-Thienyl$\mathrm{CH}), 125.1,125.3,126.9$ (ArCH), 140.4 (ArC), 169.7, 174.4, $175.7\left(\mathrm{CO}_{2} \mathrm{Me}\right.$ and CON). MS (EI) m/z (\%): 294 (M+21.72\%), 235 (53), 233 (19), 184 (10), 183 (100), 151 (54), 150 (36), 148 (14), 123 (74), 117 (13), 96 (19). HRMS calcd. for $\mathrm{C}_{13} \mathrm{H}_{14} \mathrm{~N}_{2} \mathrm{O}_{4} \mathrm{~S}$ : 294.0674; found: 294.0678 .

\section{Methyl (1S,3R,3aS,6aR)-1,5-dimethyl-4,6-dioxo-3-phenyloctahydropyrrolo[3,4-} c]pyrrole-1-carboxylate (9):<smiles>CC(=O)[C@]1(C)N[C@H](c2ccccc2)[C@H]2C(=O)N(C)C(=O)[C@H]21</smiles>

Colorless prisms, mp $=242-244{ }^{\circ} \mathrm{C}\left(\mathrm{CH}_{2} \mathrm{Cl}_{2} / \mathrm{Hex}\right) \cdot[\alpha]_{\mathrm{D}}{ }^{20}=+79.4\left(c 1, \mathrm{CHCl}_{3}\right) .72 \%$ ee from HPLC (Chiralpak AS, $1 \mathrm{~mL} / \mathrm{min}, n$-hexane/ $i$-PrOH: 90/10, $\lambda 215 \mathrm{~nm}$ ), $\mathrm{t}_{\mathrm{Rmin}}=21.3$ $\min , \mathrm{t}_{\mathrm{Rmaj}}=25.5 \mathrm{~min} . R_{\mathrm{f}}: 0.42$ (n-hexane/ethyl acetate: 1/5). IR (KBr) v. 1738, 1695, $3440 \mathrm{~cm}^{-1} .{ }^{1} \mathrm{H}$ RMN $\delta_{\mathrm{H}}: 1.61$ (s, 3H,CCH $), 2.55$ (d, $\left.J=7.9 \mathrm{~Hz}, 1 \mathrm{H}, \mathrm{NH}\right), 2.81(\mathrm{~s}, 3 \mathrm{H}$, $\mathrm{NCH}_{3}$ ), 3.27 (d, $J=7.5 \mathrm{~Hz}, 1 \mathrm{H}, \mathrm{CCH}$ ), 3.54 (dd, $J=8.8,7.7 \mathrm{~Hz}, 1 \mathrm{H}, \mathrm{CHCHCO}_{2} \mathrm{CH}_{3}$ ), 3.88 (s, 3H, $\mathrm{CO}_{2} \mathrm{CH}_{3}$ ), 4.77 (dd, $J=8.4,8.6 \mathrm{~Hz}, 1 \mathrm{H}, \mathrm{CHPh}$ ), 7.29-7.37 (m, 5H, ArH. ${ }^{13} \mathrm{C} \mathrm{RMN} \delta_{\mathrm{C}}$ : , $23.8\left(\mathrm{CCH}_{3}\right), 24.8\left(\mathrm{NCH}_{3}\right), 50.3$, 52.6 $(2 \mathrm{CHCON}), 55.6\left(\mathrm{CO}_{2} \mathrm{CH}_{3}\right), 62.1$ $(\mathrm{PhCH}), 67.2\left(\mathrm{CCH}_{3}\right), 126.9,128.3,128.4(\mathrm{ArCH}), 136.9(\mathrm{ArC}), 172.8,174.6,175.8$ (CO $\mathrm{CO}_{2} \mathrm{Me}$ and CON). MS (EI) $\mathrm{m} / z$ (\%): $302\left(\mathrm{M}^{+}, 1.35 \%\right), 244$ (16), 243 (100), 191 (53), 158 (23), 131 (41), 130 (14). HRMS calcd. for $\mathrm{C}_{16} \mathrm{H}_{18} \mathrm{~N}_{2} \mathrm{O}_{4}$ : 302.1267; found: 302.1269.

\section{Methyl (1S,3R,3aS,6aR)-1-benzyl-5-methyl-4,6-dioxo-3-} phenyloctahydropyrrolo[3,4-c]pyrrole-1-carboxylate (10):<smiles>COC(=O)[C@]1(Cc2ccccc2)N[C@@H](c2ccccc2)[C@H]2C(=O)N(C)C(=O)[C@H]21</smiles>

Colorless prisms, $\mathrm{mp}=186-188^{\circ} \mathrm{C}\left(\mathrm{CH}_{2} \mathrm{Cl}_{2} / \mathrm{Hex}\right) \cdot[\alpha]_{\mathrm{D}}{ }^{20}=-74.2\left(c 0.8, \mathrm{CHCl}_{3}\right) .98 \%$ ee from HPLC (Chiralcel ODH, $1 \mathrm{~mL} / \mathrm{min}, n$-hexane $/ i-\mathrm{PrOH}: 70 / 30, \lambda 215 \mathrm{~nm}$ ), $\mathrm{t}_{\mathrm{Rmaj}}=$ $15.6 \mathrm{~min}, \mathrm{t}_{\mathrm{Rmin}}=24.6 \mathrm{~min} . R_{\mathrm{f}}: 0.37$ (n-hexane/ethyl acetate: $\left.2 / 3\right)$. IR $(\mathrm{KBr}) v .1751$, 1696, $3377 \mathrm{~cm}^{-1} .{ }^{1} \mathrm{H}$ RMN $\delta_{\mathrm{H}}: 2.40$ (br s, $\left.1 \mathrm{H}, \mathrm{NH}\right), 2.80\left(\mathrm{~s}, 3 \mathrm{H}, \mathrm{NCH}_{3}\right), 3.06(\mathrm{~d}, J=$ $13.6 \mathrm{~Hz}, 1 \mathrm{H}, \mathrm{CH}_{2}$ ), 3.41 (d, $\left.J=7.5 \mathrm{~Hz}, 1 \mathrm{H}, \mathrm{CCH}\right)$, ), 3.46 (d, $J=13.6 \mathrm{~Hz}, 1 \mathrm{H}, \mathrm{CH}_{2}$ ), 3.56 (dd, $J=9.1,7.6 \mathrm{~Hz}, 1 \mathrm{H}, \mathrm{CHCHCO}_{2} \mathrm{CH}_{3}$ ), 3.88 (s, 3H, $\mathrm{CO}_{2} \mathrm{CH}_{3}$ ), 4.77 (d, $J=9.2$ $\mathrm{Hz}, 1 \mathrm{H}, \mathrm{CHPh}), 7.10-7.13$ (m, 2H, ArH), 7.24-7.38 (m, 8H, ArH). ${ }^{13} \mathrm{C}$ RMN $\delta_{\mathrm{C}}: 24.8$ $\left(\mathrm{NCH}_{3}\right), 40.4\left(\mathrm{CH}_{2}\right), 49.5,52.3(2 \mathrm{CHCON}), 54.2\left(\mathrm{CO}_{2} \mathrm{CH}_{3}\right), 61.1(\mathrm{PhCH}), 71.1$ $\left(\mathrm{CCH}_{2} \mathrm{Ph}\right), 127.2,127.5,128.4,128.5$ 128.9, 129.5 (ArCH), 135.0, 137.6 (ArC), 171.3, 174.6, $175.6\left(\mathrm{CO}_{2} \mathrm{Me}\right.$ and CON). MS (EI) $m / z(\%): 378\left(\mathrm{M}^{+}, 0.03 \%\right), 288$ (18), 287 (100), 142 (10). HRMS calcd. for $\mathrm{C}_{22} \mathrm{H}_{22} \mathrm{~N}_{2} \mathrm{O}_{4}$ : 378,1580; found: 378.1579 .

Methyl (1S,3R,3aS,6aR)-1-isobutyl-5-methyl-4,6-dioxo-3-(2thienyl)octahydropyrrolo[3,4-c]pyrrole-1-carboxylate (11): 


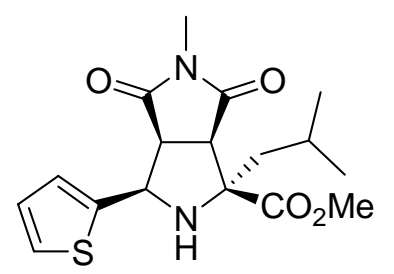

Colorless prisms, mp $=218-219^{\circ} \mathrm{C}\left(\mathrm{CH}_{2} \mathrm{Cl}_{2} / \mathrm{Hex}\right) \cdot[\alpha]_{\mathrm{D}}{ }^{20}=+18.1\left(c 0.8, \mathrm{CHCl}_{3}\right) .74 \%$ ee from HPLC (Chiralpak ODH, $1 \mathrm{~mL} / \mathrm{min}, n$-hexane $/ i$-PrOH: 90/10, $\lambda 220 \mathrm{~nm}$ ), $\mathrm{t}_{\mathrm{Rmin}}=$ $11.2 \mathrm{~min}, \mathrm{t}_{\mathrm{Rmaj}}=22.9 \mathrm{~min} . R_{\mathrm{f}}: 0.40$ (n-hexane/ethyl acetate: 2/3). IR (KBr) $v .1779$, 1712, $3338 \mathrm{~cm}^{-1} .{ }^{1} \mathrm{H}$ RMN $\delta_{\mathrm{H}}: 0.86\left(\mathrm{~d}, J=6.5 \mathrm{~Hz}, 3 \mathrm{H}, \mathrm{CHCH}_{3}\right), 0.99(\mathrm{~d}, J=6.5 \mathrm{~Hz}$, $3 \mathrm{H}, \mathrm{CHCH}_{3}$ ), $1.66-1.75$ (m, 2H, $\left.\mathrm{CH}_{2} \mathrm{CH}\right), 1.98-2.06$ (m, $1 \mathrm{H}, \mathrm{CH}_{2} \mathrm{CH}$ ), (d, $J=7.1 \mathrm{~Hz}$, $1 \mathrm{H}, \mathrm{NH}$ ), 2.84 (s, 3H, $\mathrm{NCH}_{3}$ ), 3.20 (d, $J=7.4 \mathrm{~Hz}, 1 \mathrm{H}, \mathrm{CCH}$ ), 3.46 (dd, $J=9.1,7.4 \mathrm{~Hz}$, $1 \mathrm{H}, \mathrm{CHCHCO}_{2} \mathrm{CH}_{3}$ ), 3.86 (s, 3H, $\mathrm{CO}_{2} \mathrm{CH}_{3}$ ), 4.89 (dd, $J=7.1,9.1 \mathrm{~Hz}, 1 \mathrm{H}, \mathrm{CHAr}$ ), 6.99 (dd, $J=5.1,3.6 \mathrm{~Hz}, 1 \mathrm{H}$, SCHCHCH), 7.05 (d, $J=3.0 \mathrm{~Hz}, 1 \mathrm{H}$, SCHCHCH), 7.24 (dd, $J$ $=5.1,1.1 \mathrm{~Hz}, 1 \mathrm{H}, \mathrm{SCHCHCH}) .{ }^{13} \mathrm{C} \mathrm{RMN} \delta_{\mathrm{C}}: 22.0\left(\mathrm{CH}_{2} \mathrm{CH}\right), 24.2\left(\mathrm{CHCH}_{3}\right), 24.3$ $\left(\mathrm{CHCH}_{3}\right), 24.8\left(\mathrm{NCH}_{3}\right), 42.9\left(\mathrm{CH}_{2}\right), 50.24(\mathrm{CHCON}), 52.21$ (2-Thienyl-CH), 55.9 $\left(\mathrm{CO}_{2} \mathrm{CH}_{3}\right)$, $57.6(\mathrm{CHCON}), 69.6\left(\mathrm{CCO}_{2} \mathrm{Me}\right), 1245.0,125.2,126.9(\mathrm{ArCH}), 141.2(\mathrm{ArC})$, 172.2, 174.4, $175.4\left(\mathrm{CO}_{2} \mathrm{Me}\right.$ and $\left.\mathrm{CON}\right) . \mathrm{MS}$ (EI) $m / z$ (\%): $350\left(\mathrm{M}^{+}, 2.80 \%\right), 294$ (23), 293 (44), 292 (19), 291 (100), 239 (14), 234 (10), 196 (41), 179 (13), 153 (23). HRMS calcd. for $\mathrm{C}_{17} \mathrm{H}_{22} \mathrm{~N}_{2} \mathrm{O}_{4} \mathrm{~S}$ : 350.1300; found: 350.1304. 
${ }^{1} \mathrm{H}$ NMR AND ${ }^{13} \mathrm{C}$ NMR SPECTRA

\section{COMPOUND 6b}
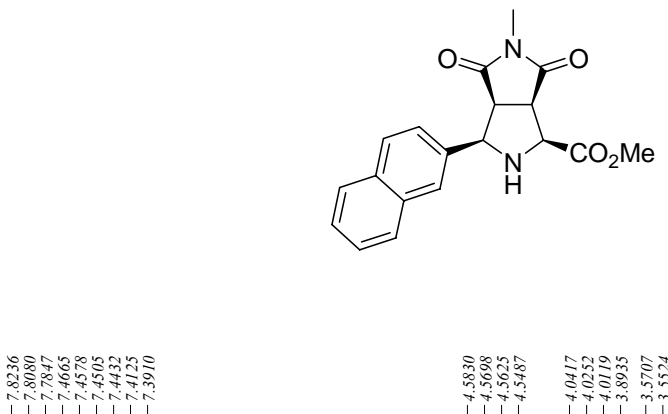

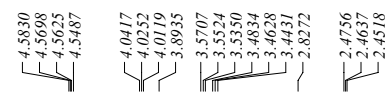
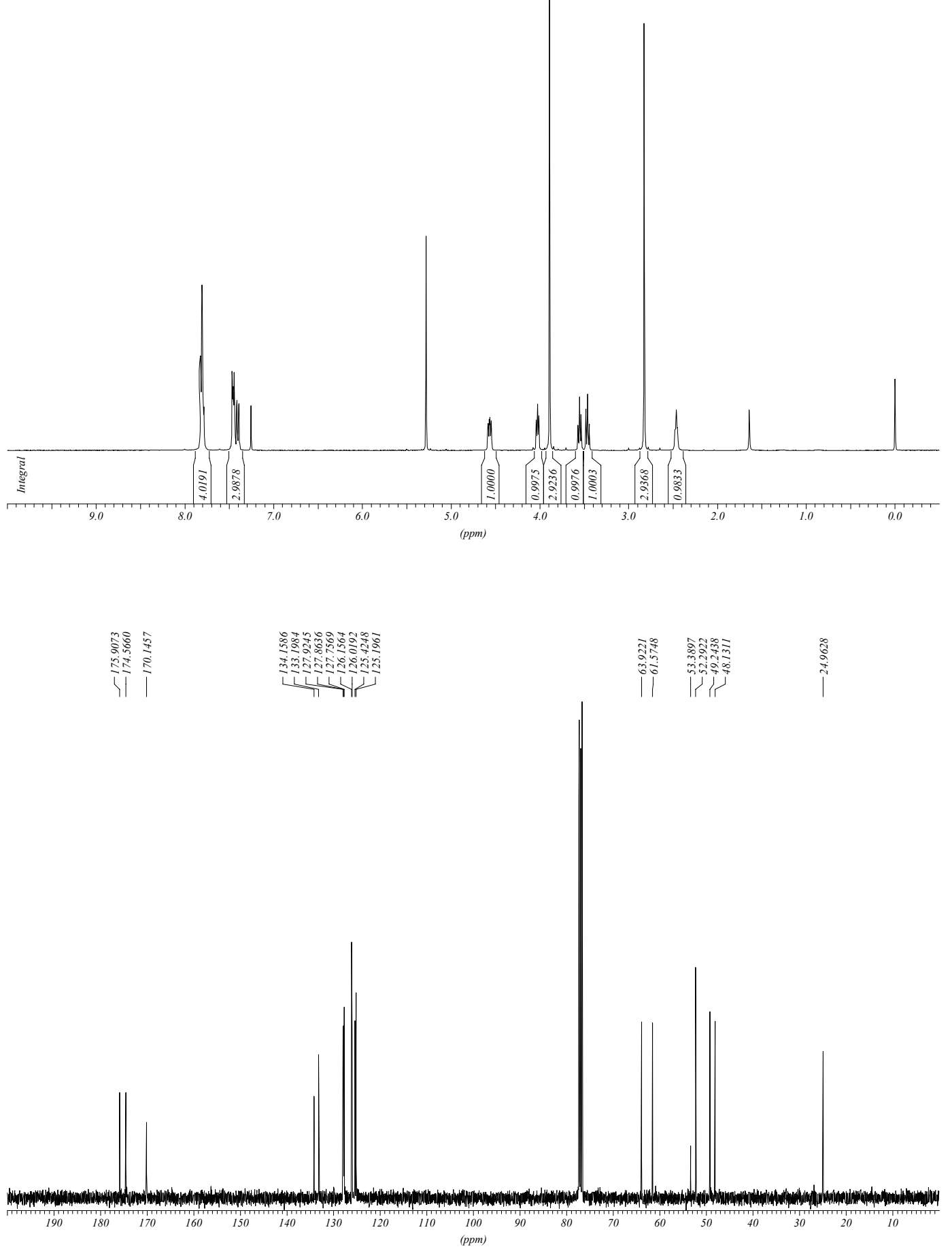
COMPOUND 6c
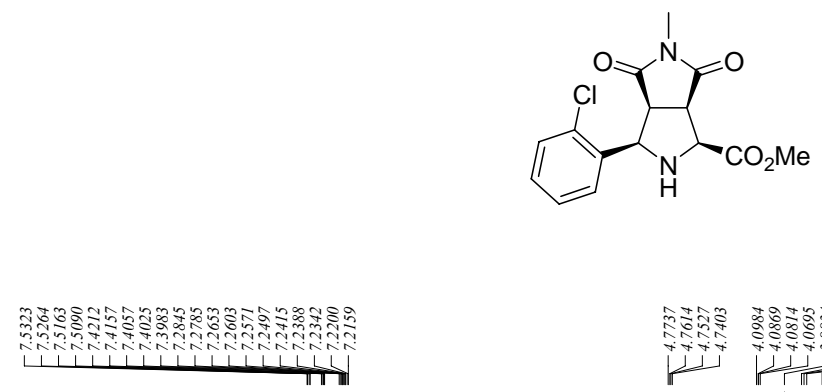

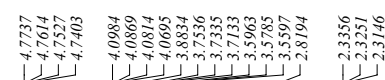

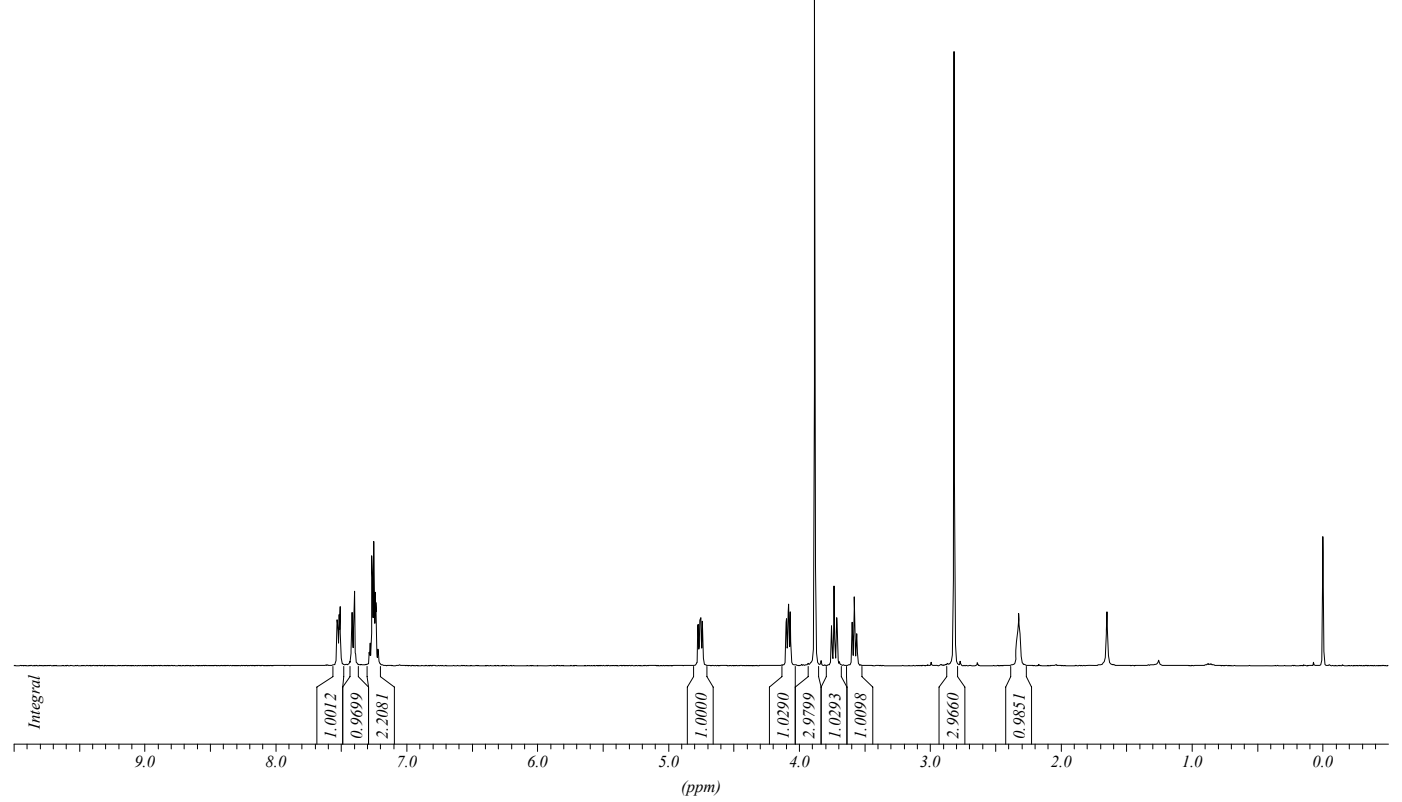

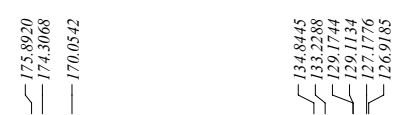

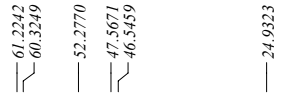

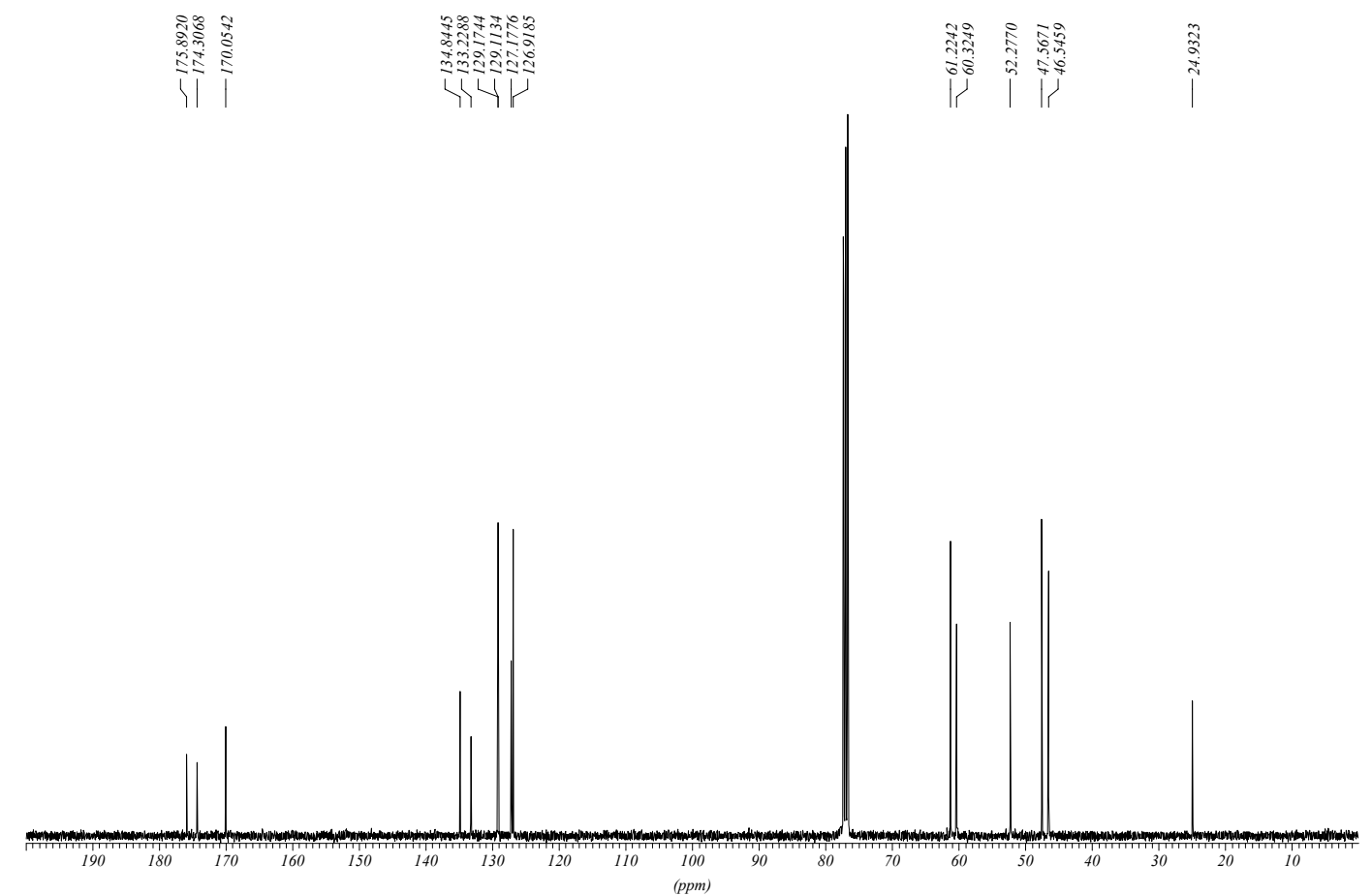




\section{COMPOUND 6d}

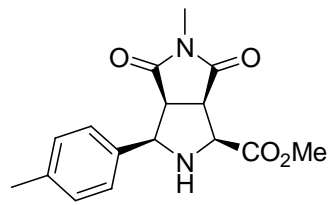

รูำำำำำ

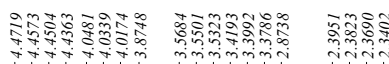

$\rightarrow$

ㄴ.t

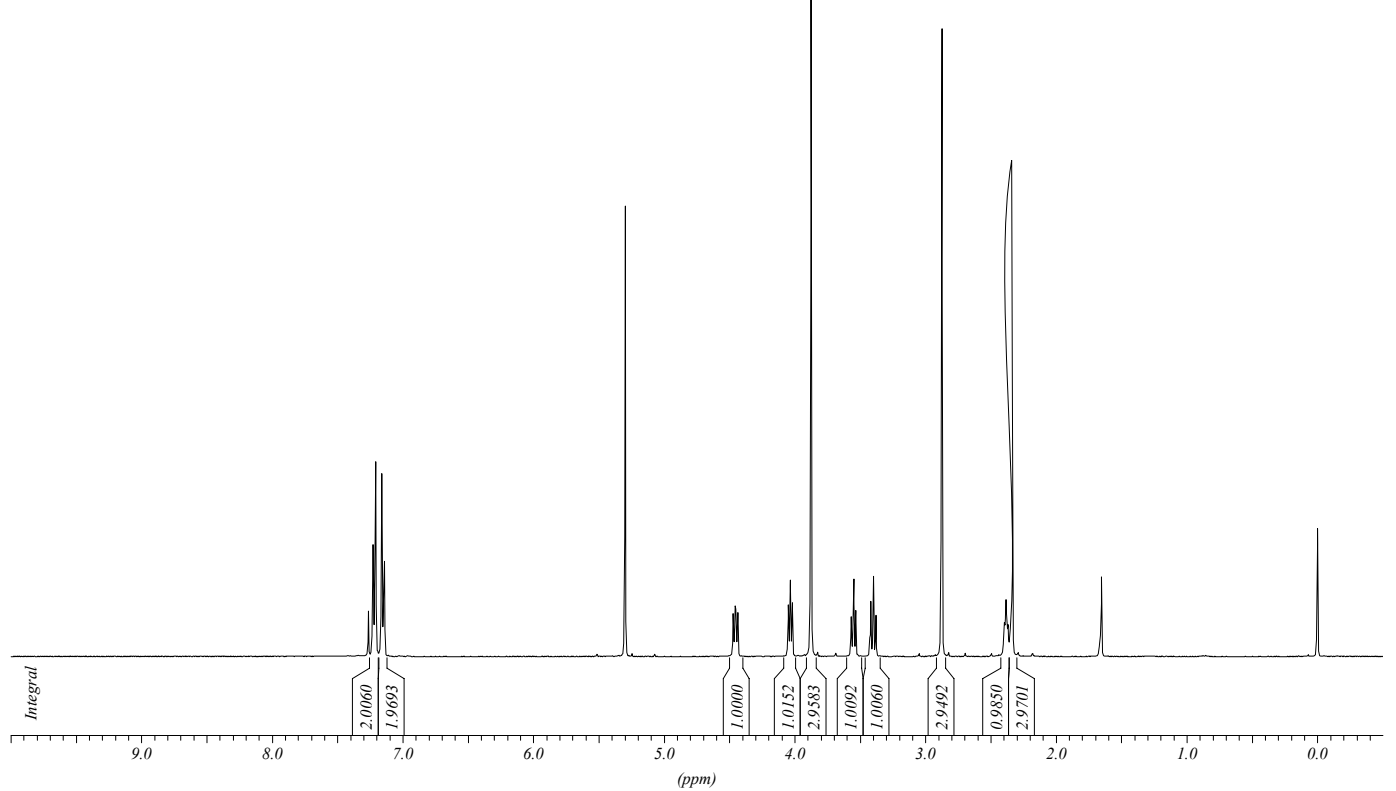

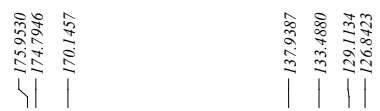

รั้ำ

iำ

iป

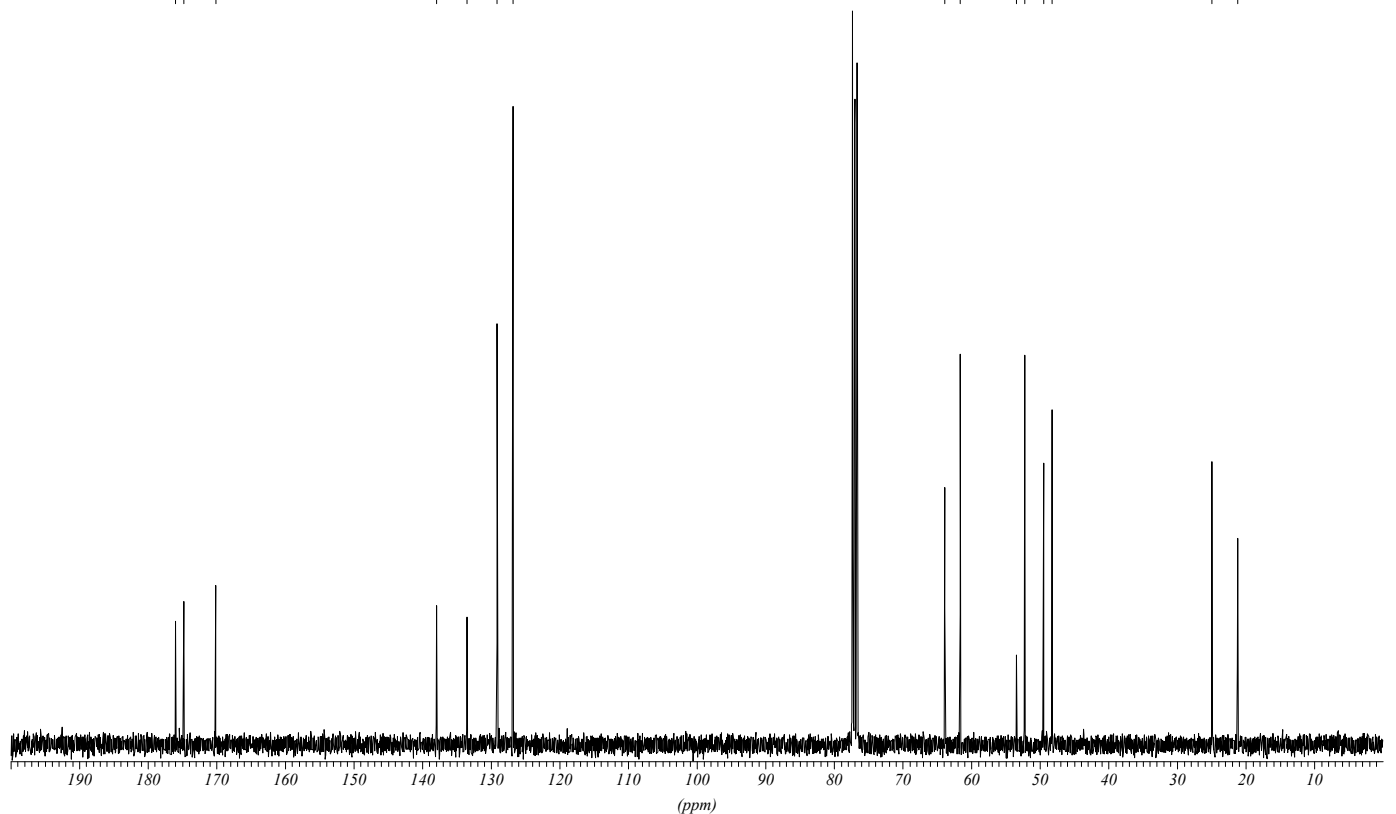


COMPOUND 6e
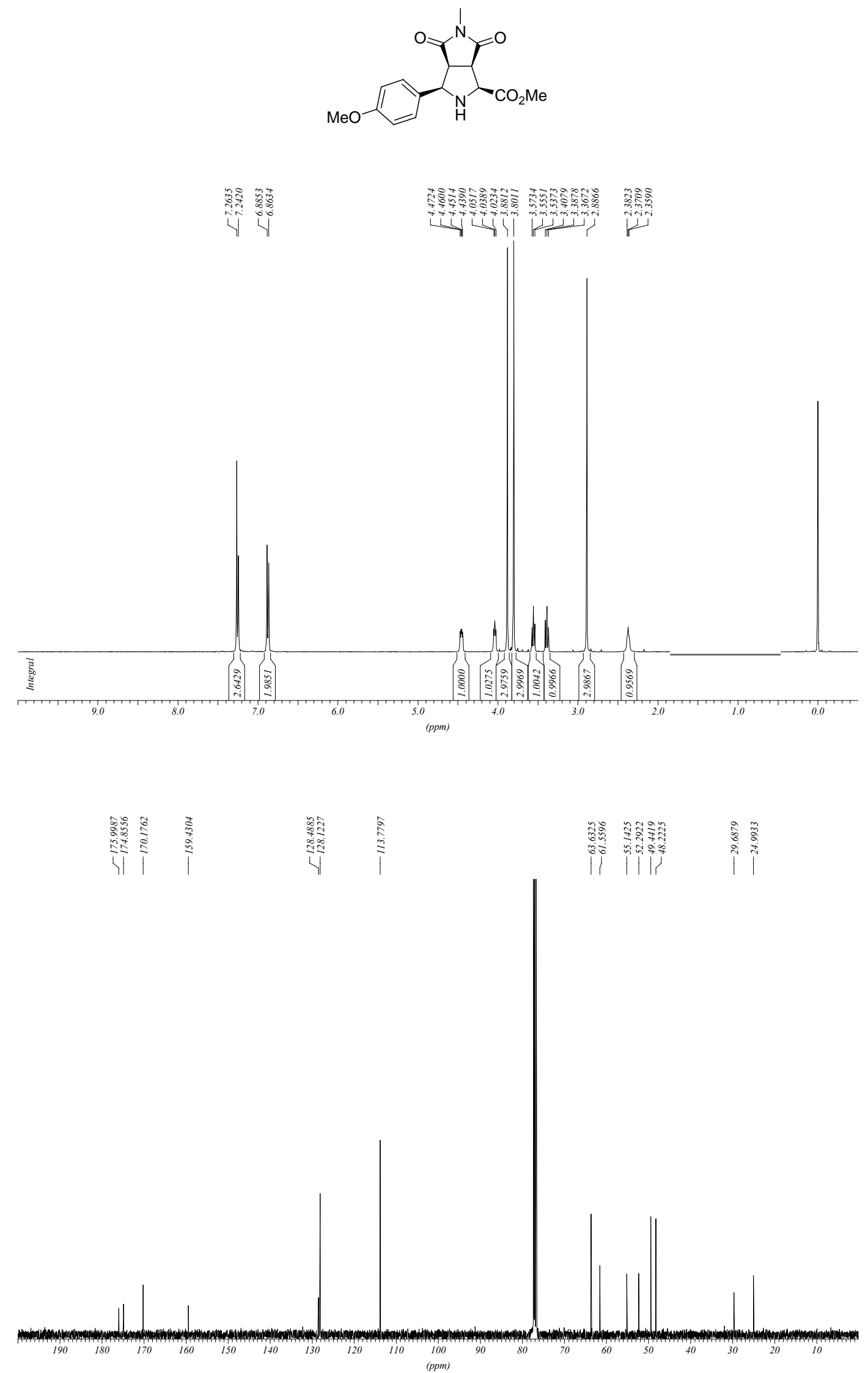


\section{COMPOUND $6 f$}
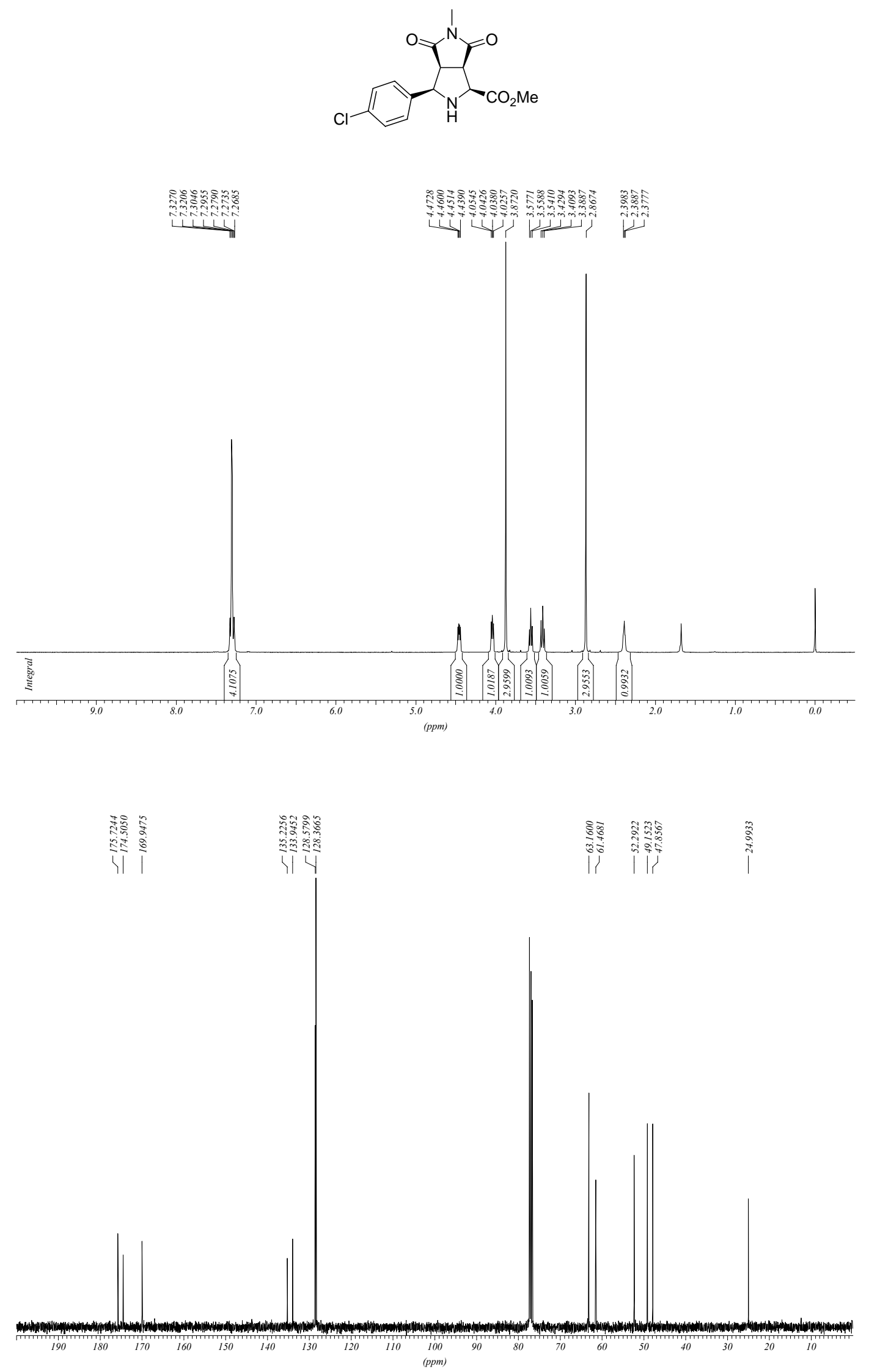
COMPOUND 6g
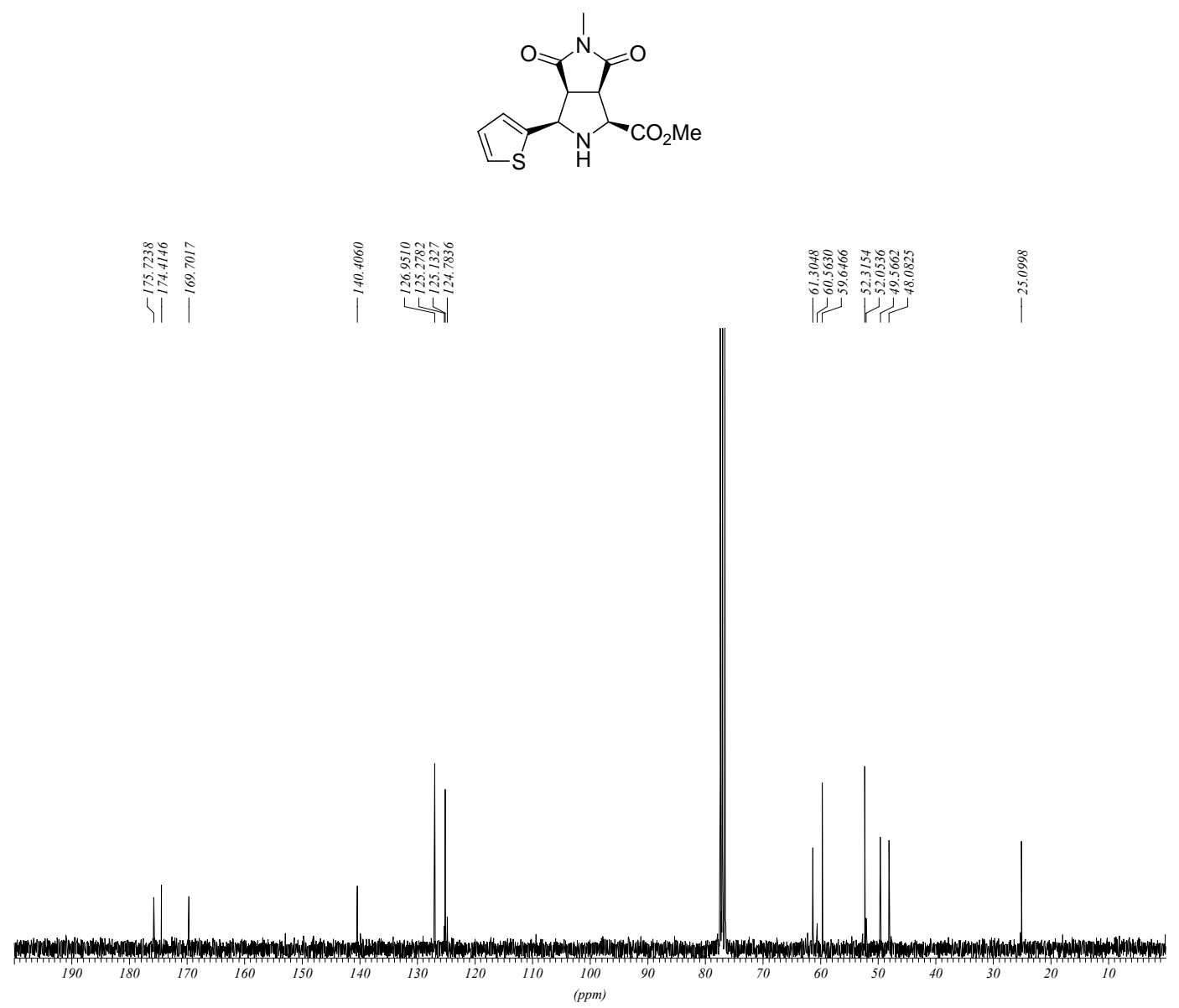

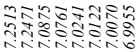

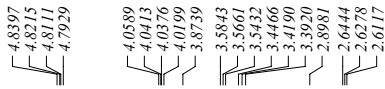

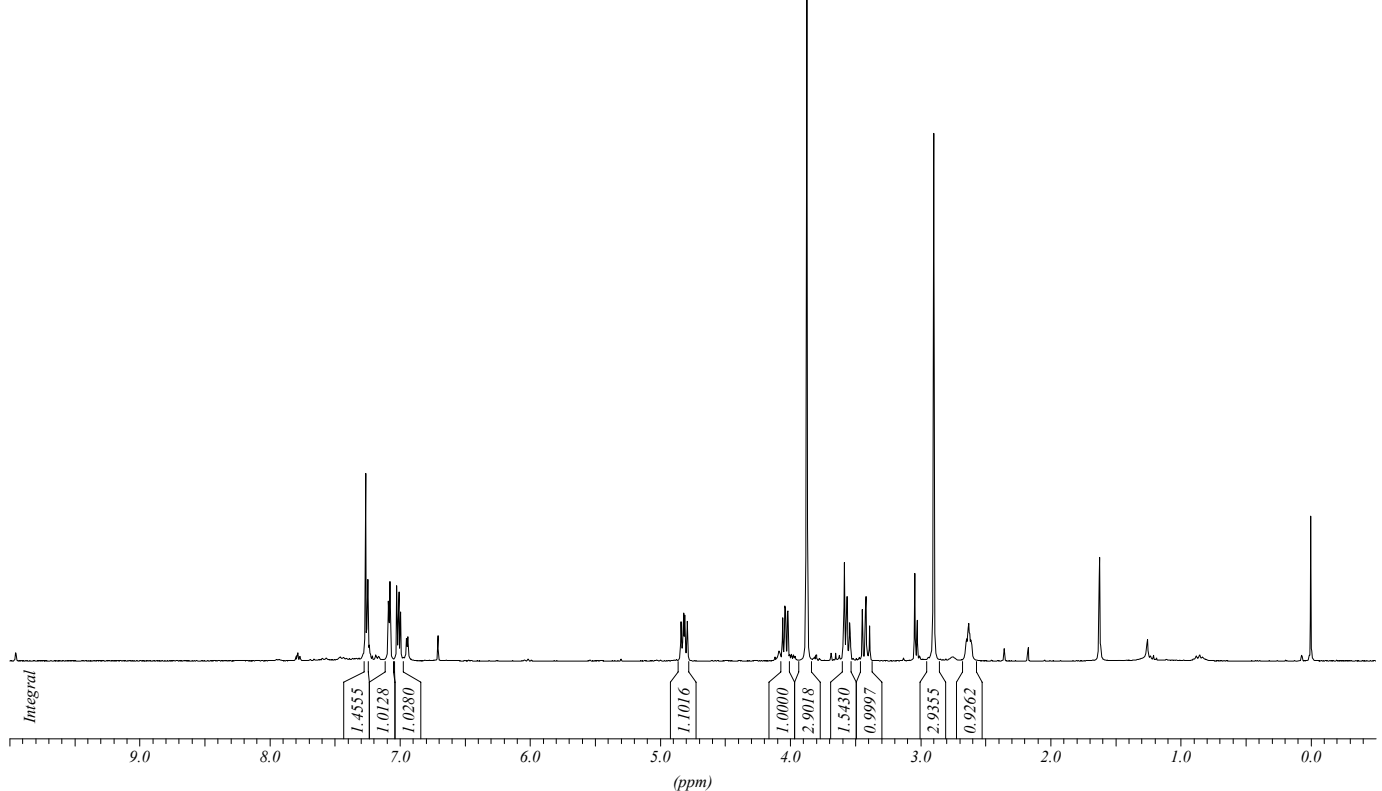


COMPOUND 9
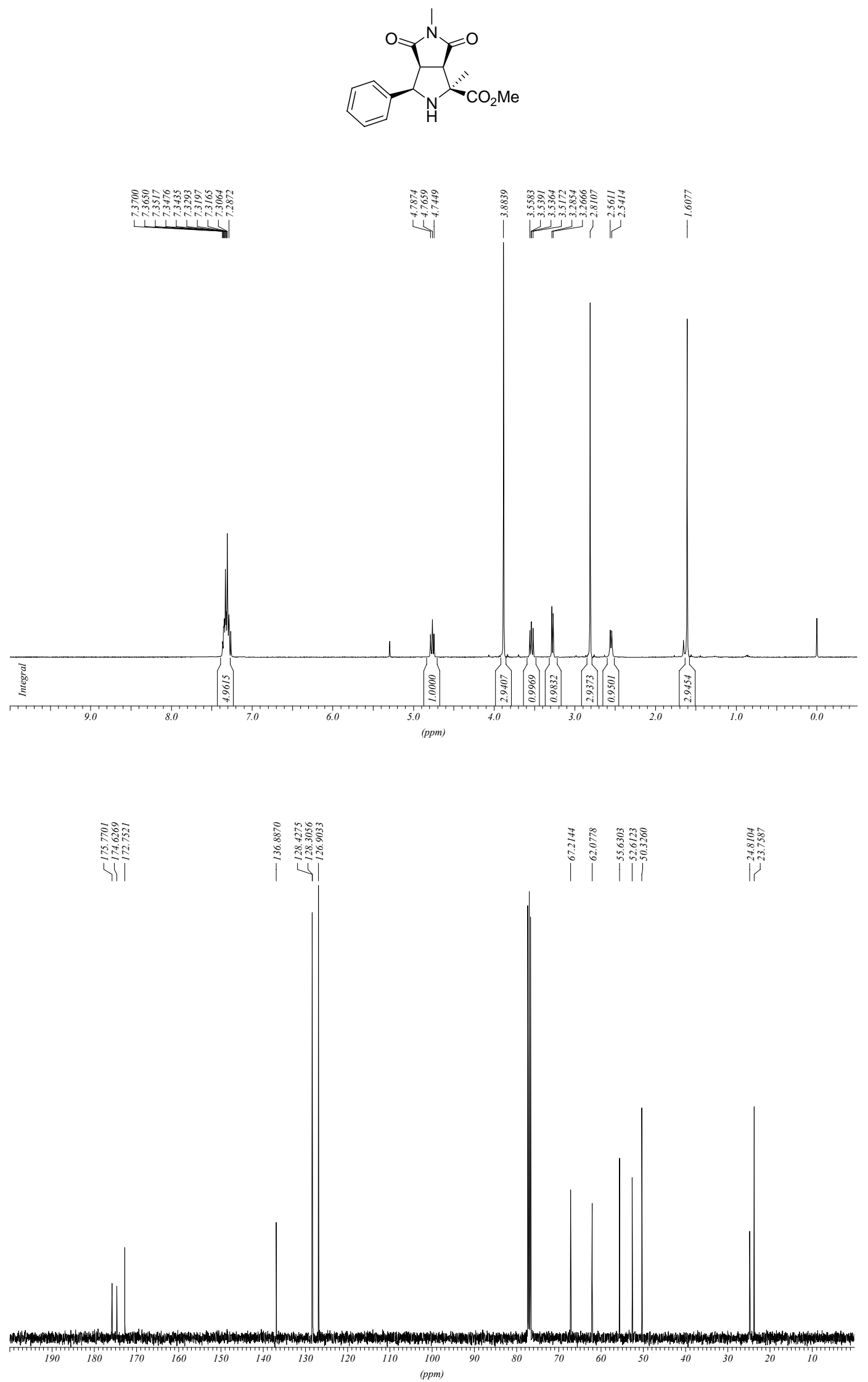
COMPOUND 10

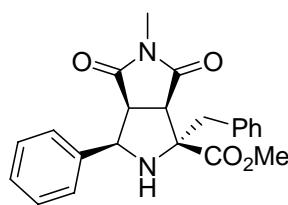

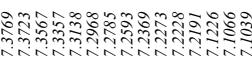

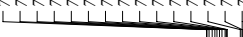

|

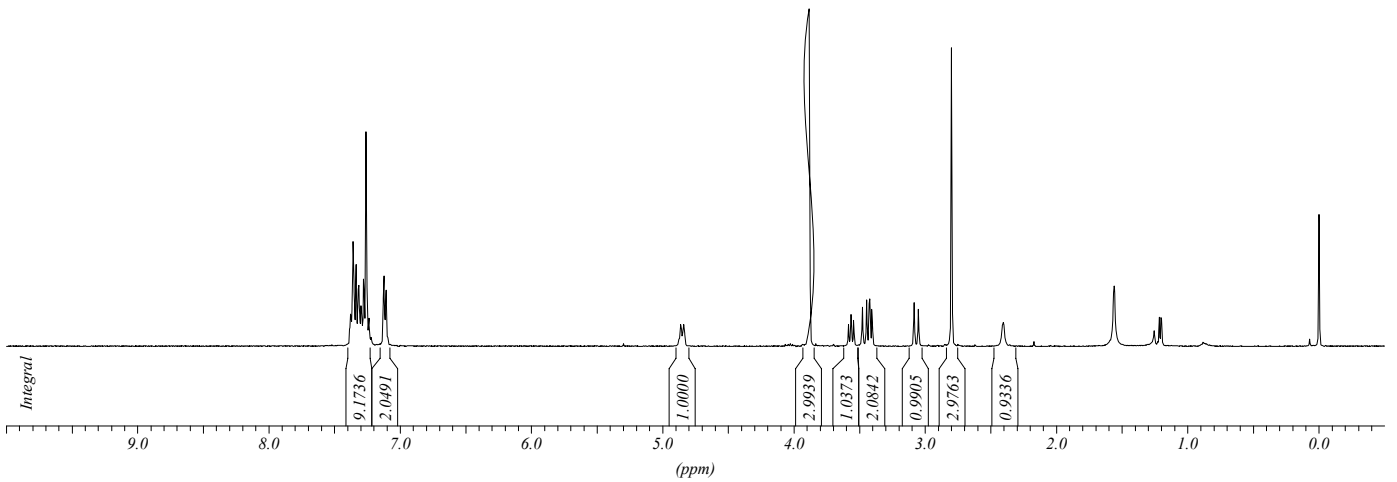

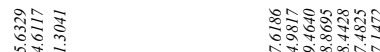

-

|

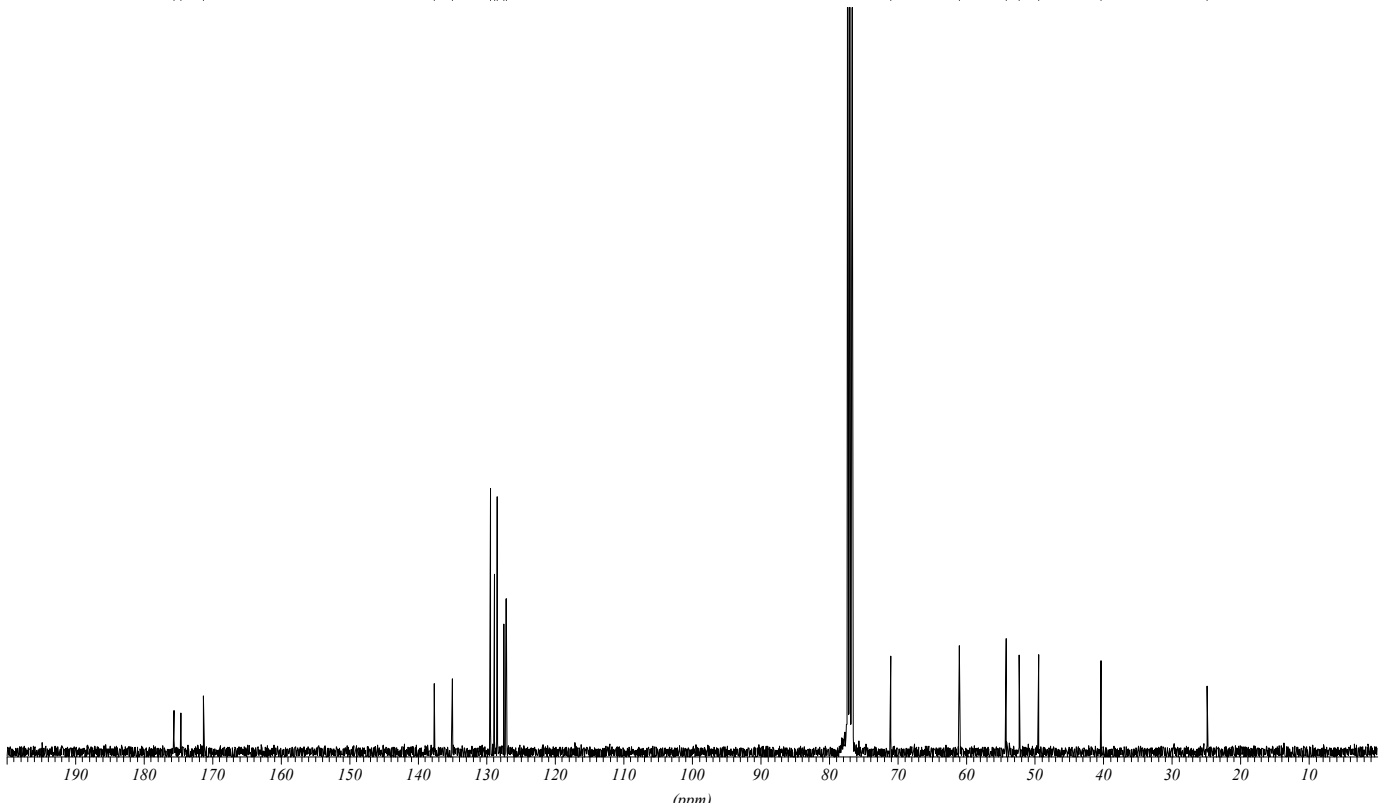


COMPOUND 11

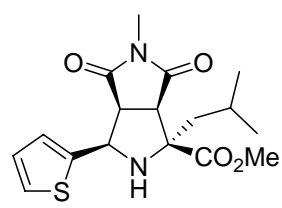

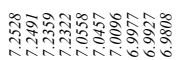

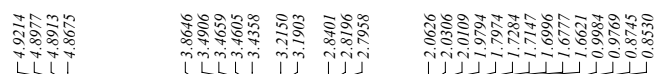

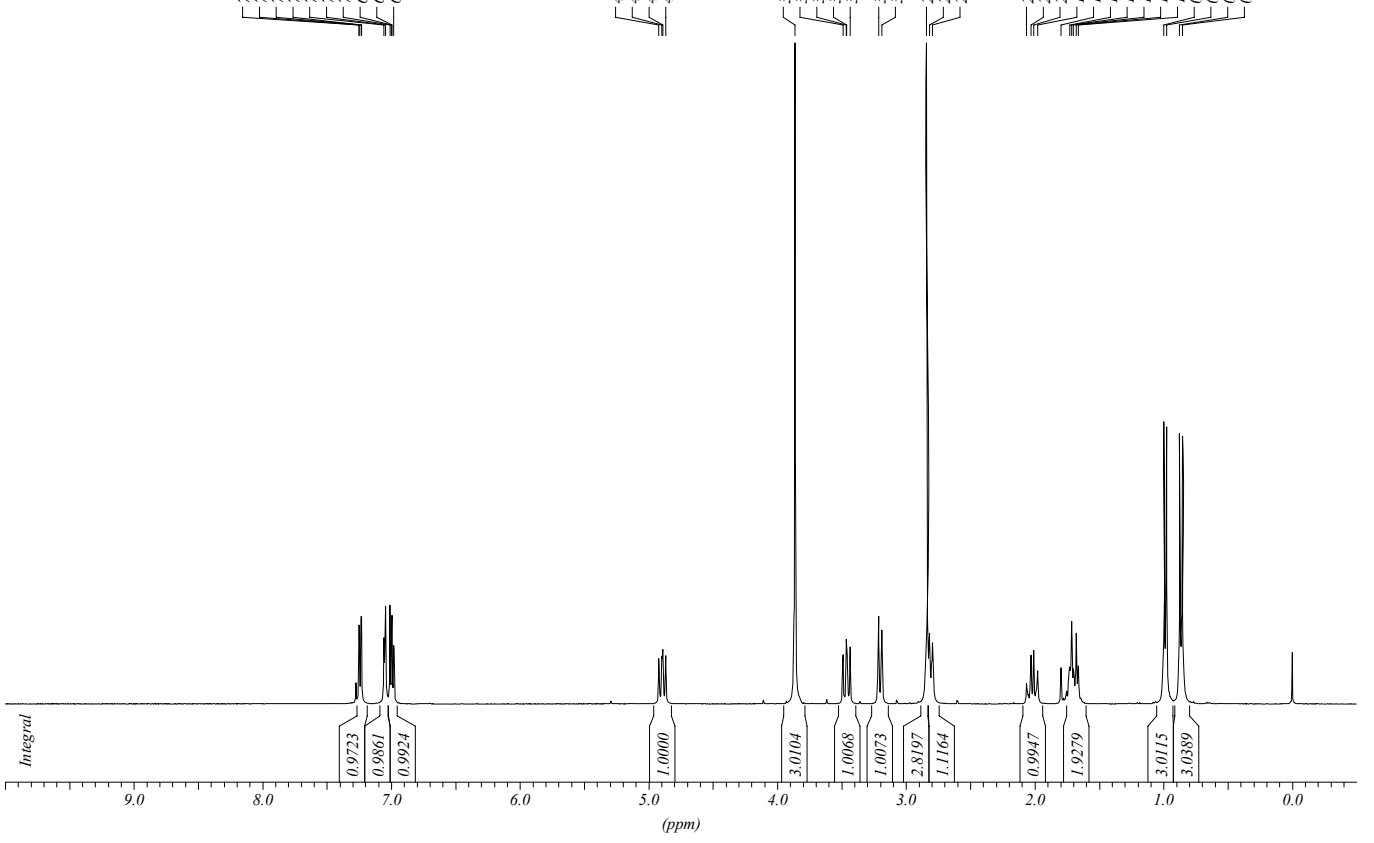

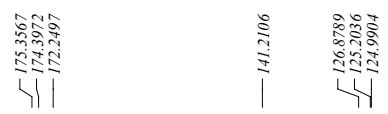

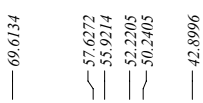

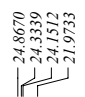

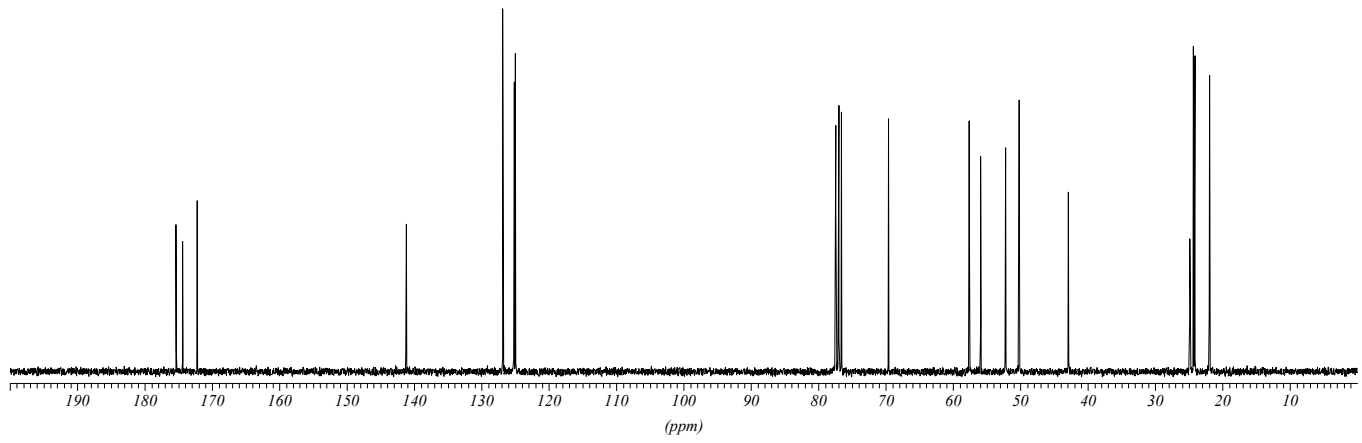




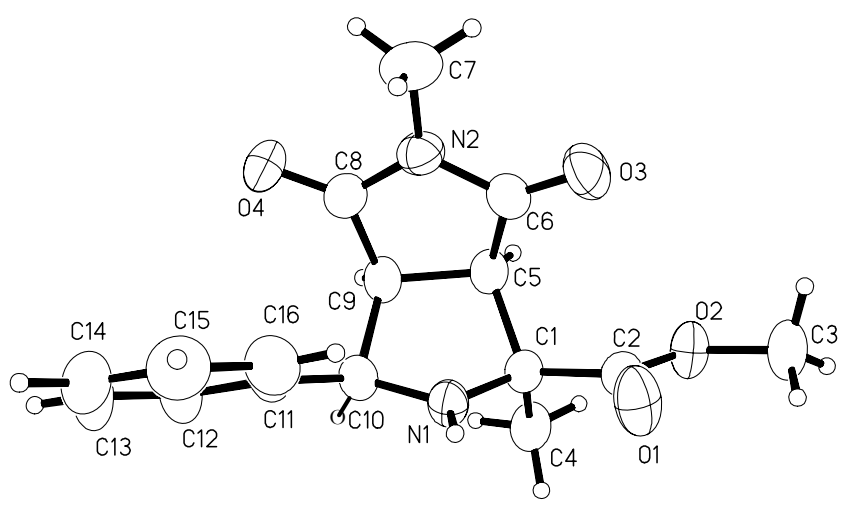

Figure 1. ORTEP drawing of compound endo-9.

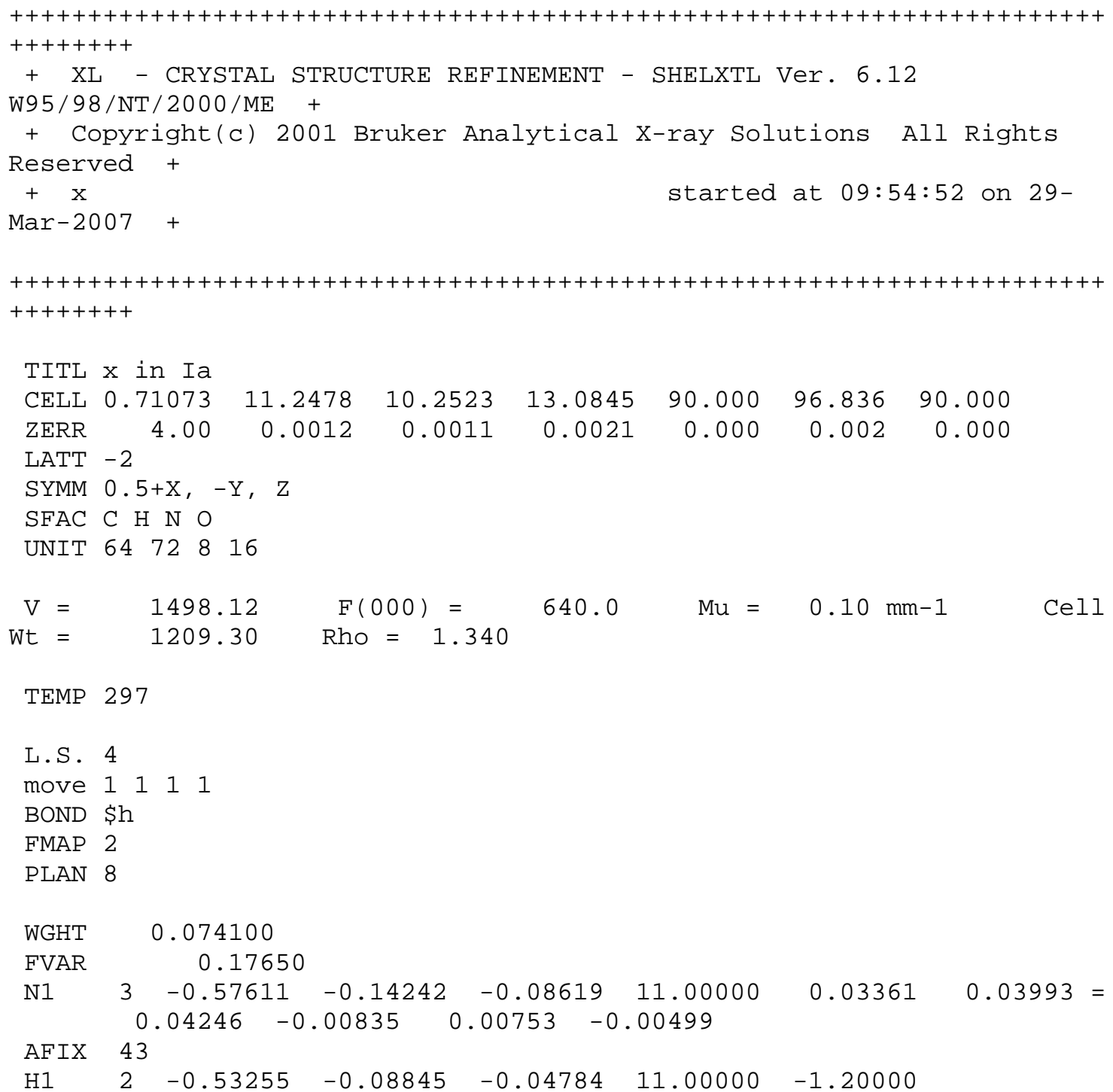


AFIX $\odot$

$\begin{array}{llllll}\text { C1 } & 1 & -0.70096 & -0.17276 & -0.07941 & 11.00000\end{array}$

$\begin{array}{llll}0.03682 & 0.00004 & 0.00695 & 0.00394\end{array}$

$\begin{array}{llllll}\text { C2 } & 1 & -0.73720 & -0.11970 & 0.02147 & 11.00000\end{array}$

$\odot .03348$

$\odot .03553$

$\begin{array}{llll}0.04212 & 0.00073 & 0.00675 & 0.00417\end{array}$

$\begin{array}{llllll}01 & 4 & -0.67210 & -0.06107 & 0.08366 & 11.00000\end{array}$ $\begin{array}{llll}0.04819 & -0.02671 & 0.01173 & -0.01722\end{array}$

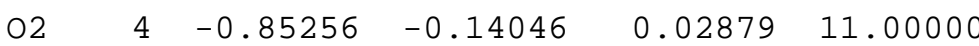
$\begin{array}{llll}0.04679 & -0.01305 & 0.00993 & 0.00290\end{array}$

C3 $\quad \begin{array}{lllll}1 & -0.89687 & -0.09391 & 0.12007 & 11.00000\end{array}$ $\begin{array}{llll}0.05254 & -0.00937 & 0.01735 & 0.00735\end{array}$

AFIX 137

\begin{tabular}{lccccc} 
H3A & 2 & -0.88956 & -0.16140 & 0.17130 & 11.00000 \\
H3B & 2 & -0.97953 & -0.06990 & 0.10449 & 11.00000 \\
H3C & 2 & -0.85125 & -0.01920 & 0.14570 & 11.00000 \\
AFIX & 0 & & & & \\
C4 & 1 & -0.78804 & -0.12065 & -0.16936 & $11.0000 \odot$ \\
& \multicolumn{2}{c}{0.03875} & 0.00203 & 0.00541 & 0.01253
\end{tabular}

AFIX 137 $\begin{array}{llll}0.03875 & 0.00203 & 0.00541 & 0.01253\end{array}$

\begin{tabular}{|c|c|c|c|c|c|}
\hline $\mathrm{H} 4 \mathrm{~A}$ & 2 & -0.78008 & -0.02762 & -0.17345 & 11.00000 \\
\hline $\mathrm{H} 4 \mathrm{~B}$ & 2 & -0.86862 & -0.14224 & -0.15844 & 11.00000 \\
\hline $\mathrm{H} 4 \mathrm{C}$ & 2 & -0.76990 & -0.15949 & -0.23244 & 11.00000 \\
\hline AFIX & 6 & & & & \\
\hline C5 & 1 & $\begin{array}{l}-0.69683 \\
03534\end{array}$ & $\begin{array}{l}-0.32357 \\
00545\end{array}$ & $\begin{array}{l}-0.08413 \\
00354\end{array}$ & $\begin{array}{l}11.0000 \odot \\
00 \odot 77\end{array}$ \\
\hline
\end{tabular}

AFIX 13

$\begin{array}{lccccc}\text { H5 } & 2 & -0.77509 & -0.36036 & -0.10993 & 11.00000\end{array}$

$\begin{array}{llllll}\text { C6 } & 1 & -0.64616 & -0.38557 & 0.01699 & 11.0000 \odot\end{array}$

$\begin{array}{llllll}0 & 0.03672 & -0.00171 & 0.00516 & -0.00504 \\ 03 & 4 & -0.68377 & -0.37588 & 0.09865 & 11.00000\end{array}$ $\begin{array}{llll}0.03866 & 0.00763 & 0.01636 & 0.00750\end{array}$

$\begin{array}{llllll}N 2 & 3 & -0.54802 & -0.46102 & 0.00012 & 11.00000\end{array}$ $\begin{array}{llll}0.04727 & 0.00483 & 0.00213 & 0.00383\end{array}$

$\begin{array}{llllll}C 7 & 1 & -0.48283 & -0.54130 & 0.07888 & 11.00000\end{array}$ $\begin{array}{llll}0.06766 & 0.01797 & 0.00290 & 0.01418\end{array}$

AFIX 137

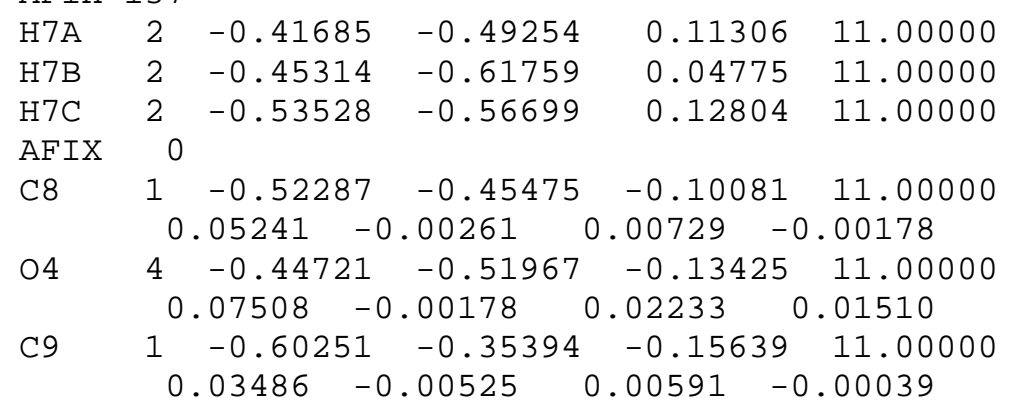

AFIX 13

$\begin{array}{llllll}\text { H9 } & 2 & -0.64007 & -0.38724 & -0.22269 & 11.00000\end{array}$

$\begin{array}{llllll}\text { C10 } & 1 & -0.53774 & -0.22026 & -0.17009 & 11.00000\end{array}$ $\begin{array}{llll}0.03758 & 0.00207 & 0.00836 & 0.00223\end{array}$

$\begin{array}{llllll}\mathrm{C} 11 & 1 & -0.40325 & -0.22828 & -0.16595 & 11.00000\end{array}$ $\begin{array}{llll}0.04824 & 0.00190 & 0.01699 & -0.00254\end{array}$

$\begin{array}{llllll}\mathrm{C} 12 & 1 & -0.35386 & -0.25276 & -0.25654 & 11.00000\end{array}$ $\begin{array}{llll}0.07029 & -0.00566 & 0.03020 & -0.00219\end{array}$

AFIX 43

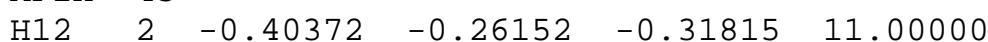

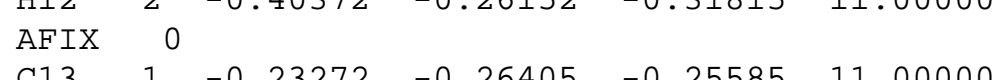

$\begin{array}{cccccc}\mathrm{C} 13 & 1 & -0.23272 & -0.26405 & -0.25585 & 11.00000 \\ 0.11387 & -0.01418 & 0.05101 & 0.00037\end{array}$

AFIX 43

$\begin{array}{lllllll}\mathrm{H} 13 & 2 & -0.20121 & -0.28132 & -0.31701 & 11.0000 \odot & -1.2000 \odot\end{array}$
$0.050810 .08427=$

$0.036620 .06295=$

$0.046050 .06899=$

$-1.50000$

$-1.50000$

$-1.50000$

$\odot .04444$

$0.05703=$

$-1.50000$

$-1.50000$

$-1.50000$

$\odot .03060$

$0.04007=$

$-1.20000$

๑. 03996

$0.04215=$

$\odot .06545$

$0.07387=$

$\odot .03958$

$0.04020=$

$\odot .07076$

$0.05788=$

$-1.50000$

$-1.50000$

$-1.50000$

๑. 03541

๑. 05196

$0.03808=$

$0.05012=$

๑. . 03273

$0.04451=$

$-1.20000$

$\odot .03503$

$0.04528=$

๑. 03954

$0.03811=$

$\odot .05506$

$0.06198=$

$-1.20000$

๑. 06324

$0.08279=$ 


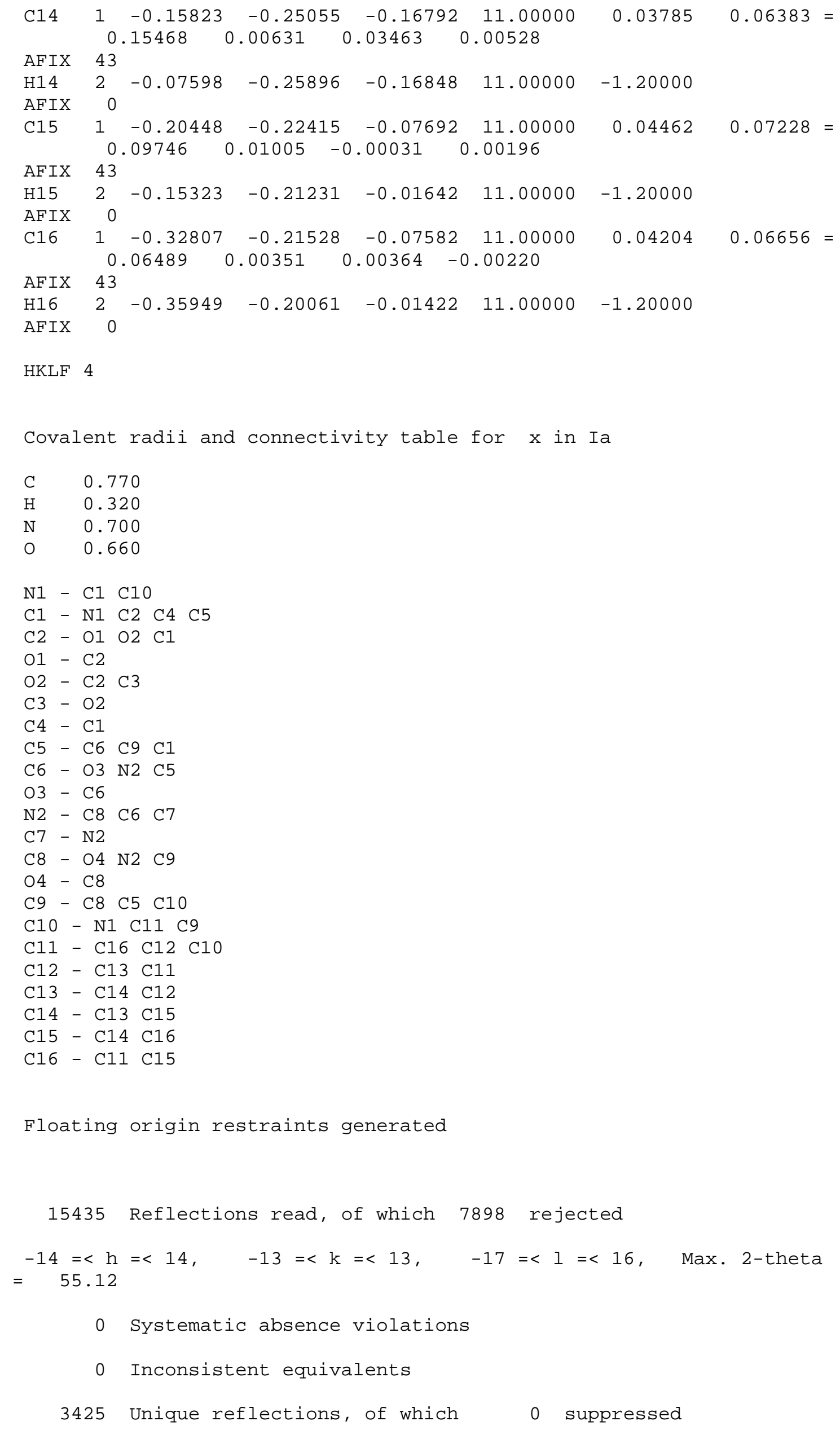

Floating origin restraints generated

15435 Reflections read, of which 7898 rejected 


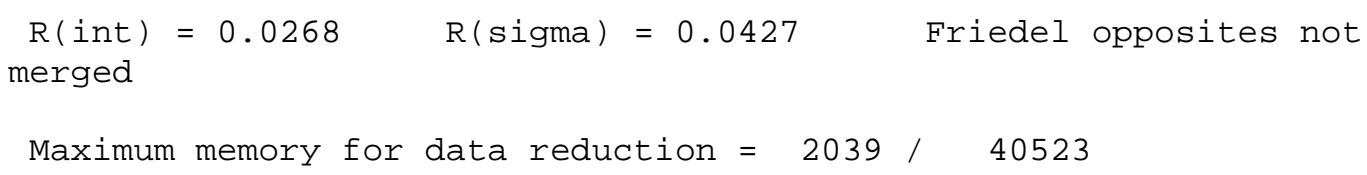




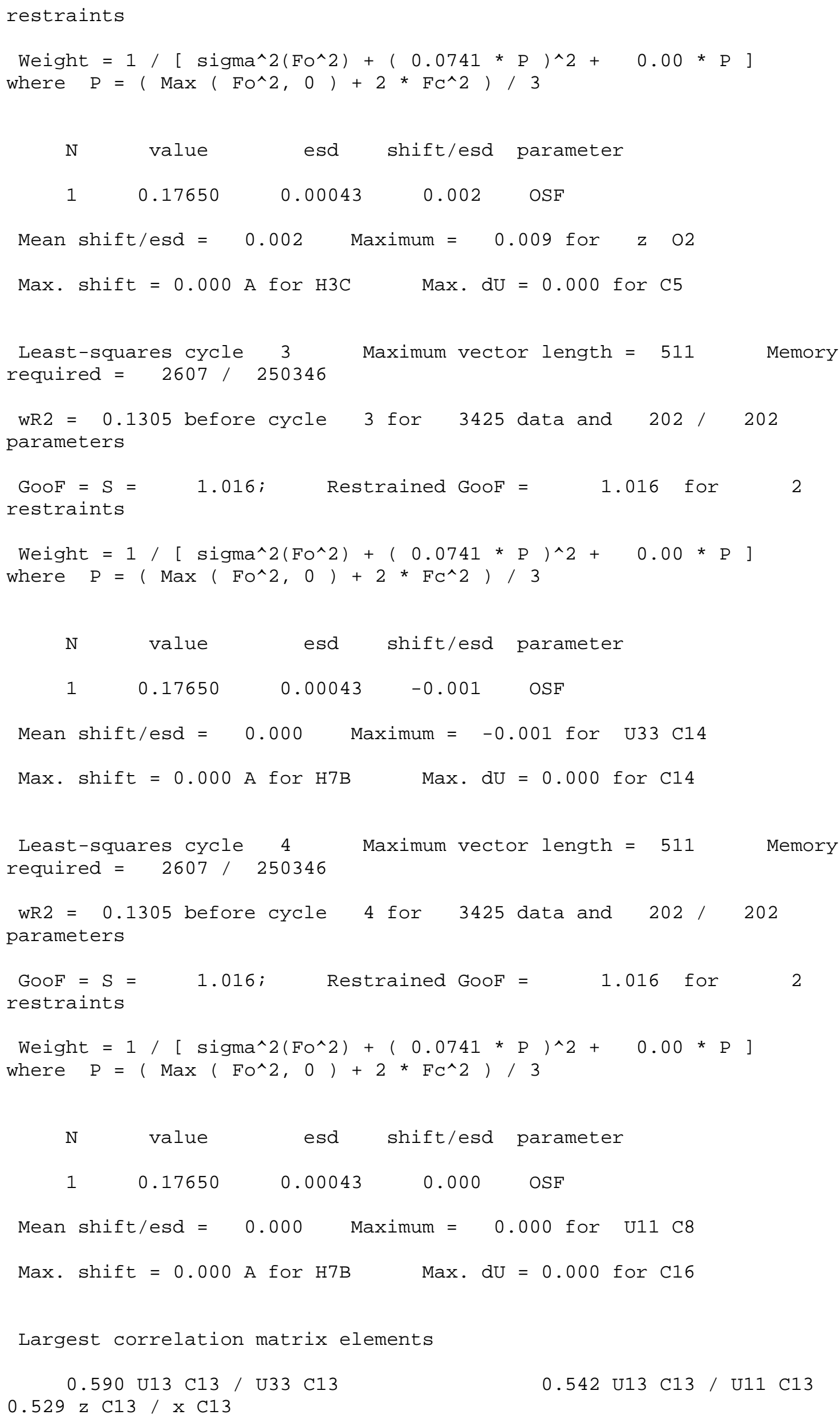


Idealized hydrogen atom generation before cycle 5

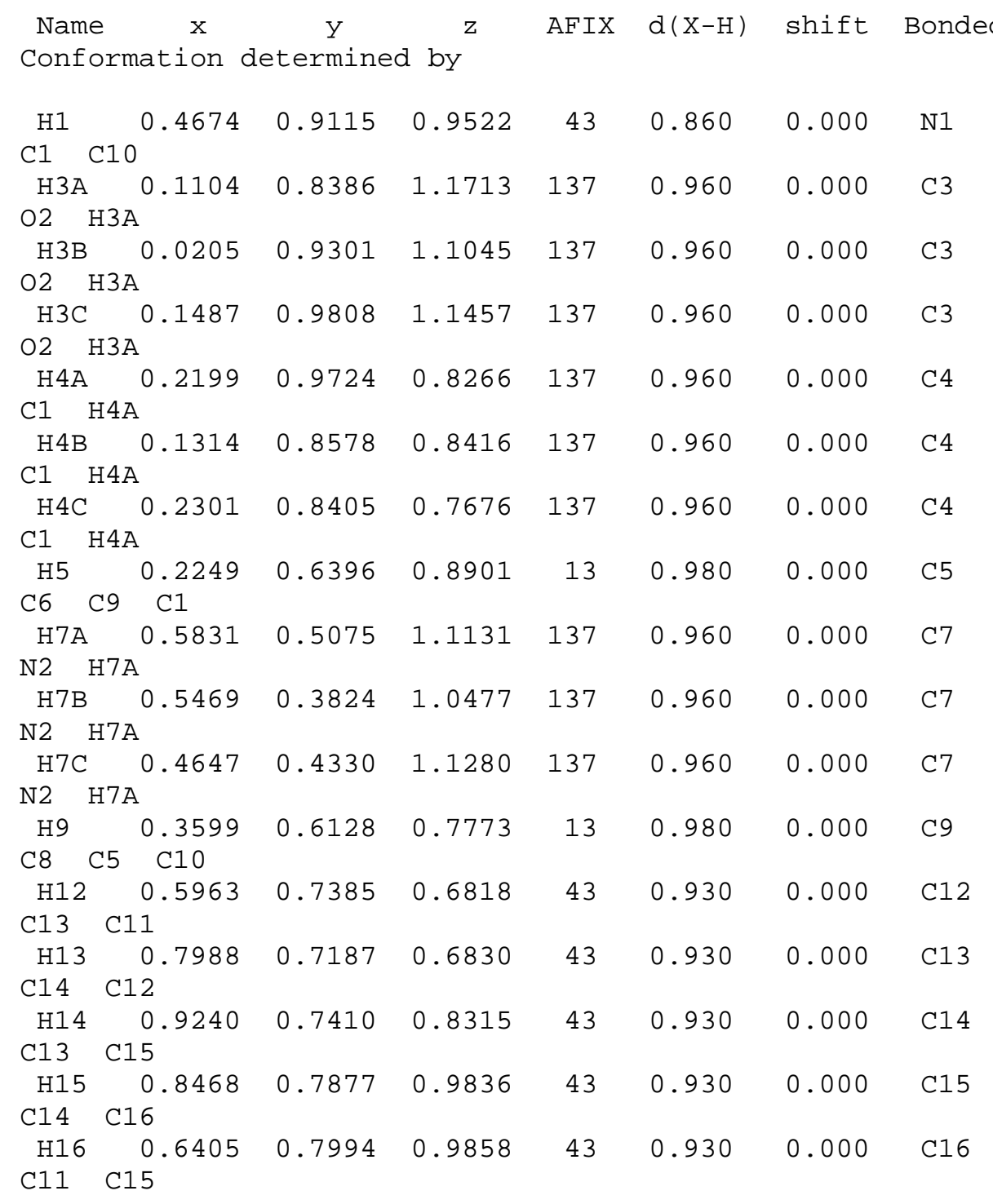

$\mathrm{x}$ in $\mathrm{Ia}$

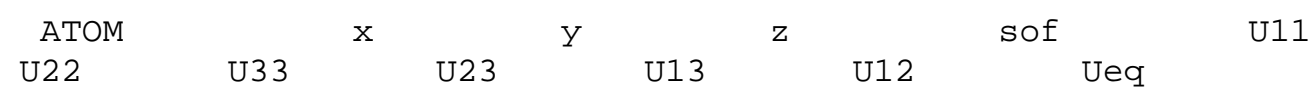
$\begin{array}{llllll}\mathrm{N} 1 & 0.42389 & 0.85758 & 0.91380 & 1.00000 & 0.03361\end{array}$
$\begin{array}{llllll}0.03993 & 0.04246 & -0.00835 & 0.00753 & -0.00499 & 0.03842\end{array}$
$\begin{array}{llllll}0.00348 & 0.00017 & 0.00018 & 0.00016 & 0.00000 & 0.00103\end{array}$
$\begin{array}{llllll}0.00096 & 0.00111 & 0.00088 & 0.00084 & 0.00082 & 0.00045\end{array}$
H1
0.46745
0.91155
$\odot .95216$
1.00000
$\odot .04611$
0.00000
0.00000
$\begin{array}{llllll}\text { C1 } & 0.29904 & 0.82724 & 0.92059 & 1.00000 & 0.03348\end{array}$

$\begin{array}{llllll}0.03919 & 0.03682 & 0.00004 & 0.00695 & 0.00394 & 0.03628\end{array}$

$\begin{array}{llllll}0.00437 & 0.00021 & 0.00023 & 0.00018 & 0.00000 & 0.00116\end{array}$

$\begin{array}{llllll}0.00114 & 0.00121 & 0.00097 & 0.00095 & 0.00093 & 0.00050\end{array}$

$\begin{array}{llllll}C 2 & 0.26280 & 0.88030 & 1.02147 & 1.00000 & 0.03553\end{array}$

$\begin{array}{llllll}0.03998 & 0.04212 & 0.00073 & 0.00675 & 0.00417 & 0.03904\end{array}$ 

0.00464
0.00021
0.00023
0.00019
$\odot .00000$
0.00121
$\begin{array}{llllll}0.00118 & 0.00136 & 0.00100 & 0.00104 & 0.00100 & 0.00053\end{array}$

\begin{tabular}{ccccccc}
01 & \multicolumn{2}{c}{0.32790} & 0.93893 & 1.08366 & \multicolumn{1}{c}{1.00000} & 0.05081 \\
0.08427 & 0.04819 & -0.02671 & 0.01173 & -0.01722 & 0.06062 \\
0.00336 & 0.00017 & 0.00021 & 0.00015 & 0.00000 & 0.00113 \\
0.00141 & 0.00112 & 0.00103 & 0.00092 & 0.00105 & 0.00057
\end{tabular}

$\begin{array}{llllll}02 & 0.14744 & 0.85954 & 1.02880 & 1.00000 & 0.03662\end{array}$

$\begin{array}{llllll}0.06295 & 0.04679 & -0.01305 & 0.00993 & 0.00290 & 0.04838\end{array}$

$\begin{array}{llllll}0.00313 & 0.00015 & 0.00018 & 0.00014 & 0.00000 & 0.00096\end{array}$ $\begin{array}{llllll}0.00114 & 0.00102 & 0.00084 & 0.00078 & 0.00080 & 0.00046\end{array}$

\begin{tabular}{lcccccc} 
C3 & 0.10313 & 0.90609 & 1.12007 & \multicolumn{2}{c}{1.00000} & 0.04605 \\
0.06899 & 0.05254 & -0.00937 & 0.01735 & 0.00735 & 0.05494 \\
0.00555 & 0.00025 & 0.00032 & 0.000022 & 0.00000 & 0.00145 \\
0.00178 & 0.00162 & 0.00139 & 0.00126 & 0.00134 & 0.00070
\end{tabular}

$\begin{array}{llllll}0.05703 & 0.03875 & 0.00203 & 0.00541 & 0.01253 & 0.04670\end{array}$

$\begin{array}{llllll}0.00513 & 0.00023 & 0.00027 & 0.00020 & 0.00000 & 0.00146\end{array}$ $\begin{array}{llllll}0.00152 & 0.00135 & 0.00110 & 0.00112 & 0.00118 & 0.00062\end{array}$

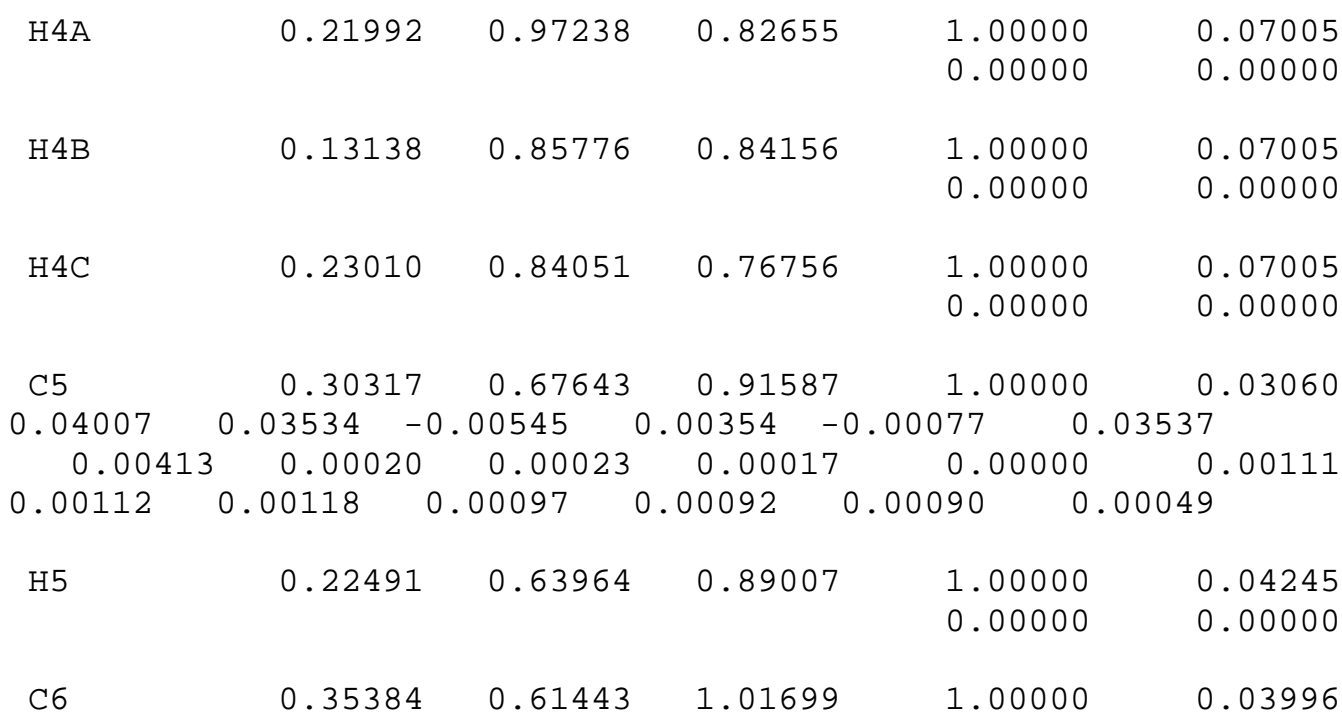

$\begin{array}{llllll}0.04215 & 0.03672 & -0.00171 & 0.00516 & -0.00504 & 0.03956\end{array}$

$\begin{array}{llllll}0.00424 & 0.00022 & 0.00024 & 0.00018 & 0.00000 & 0.00126\end{array}$

$\begin{array}{llllll}0.00122 & 0.00132 & 0.00102 & 0.00103 & 0.00105 & 0.00054\end{array}$
03
$\begin{array}{lll}0.31623 & 0.62412 & 1.09864\end{array}$
1.00000
$\odot .06545$
$\begin{array}{llllll}0.07387 & 0.03866 & 0.00763 & 0.01636 & 0.00750 & 0.05851\end{array}$
$\begin{array}{llllll}0.00395 & 0.00019 & 0.00021 & 0.00015 & 0.00000 & 0.00132\end{array}$
$\begin{array}{llllll}0.00126 & 0.00100 & 0.00090 & 0.00093 & 0.00102 & 0.00052\end{array}$
N2
$0.45198 \quad 0.53898 \quad 1.00012$
1.00000
0.03958
$\begin{array}{llllll}0.04020 & 0.04727 & 0.00483 & 0.00213 & 0.00383 & 0.04259\end{array}$
$\begin{array}{llllll}0.00370 & 0.00019 & 0.00018 & 0.00016 & 0.00000 & 0.00111\end{array}$
$\begin{array}{llllll}0.00109 & 0.00126 & 0.00092 & 0.00095 & 0.00091 & 0.00050\end{array}$ 


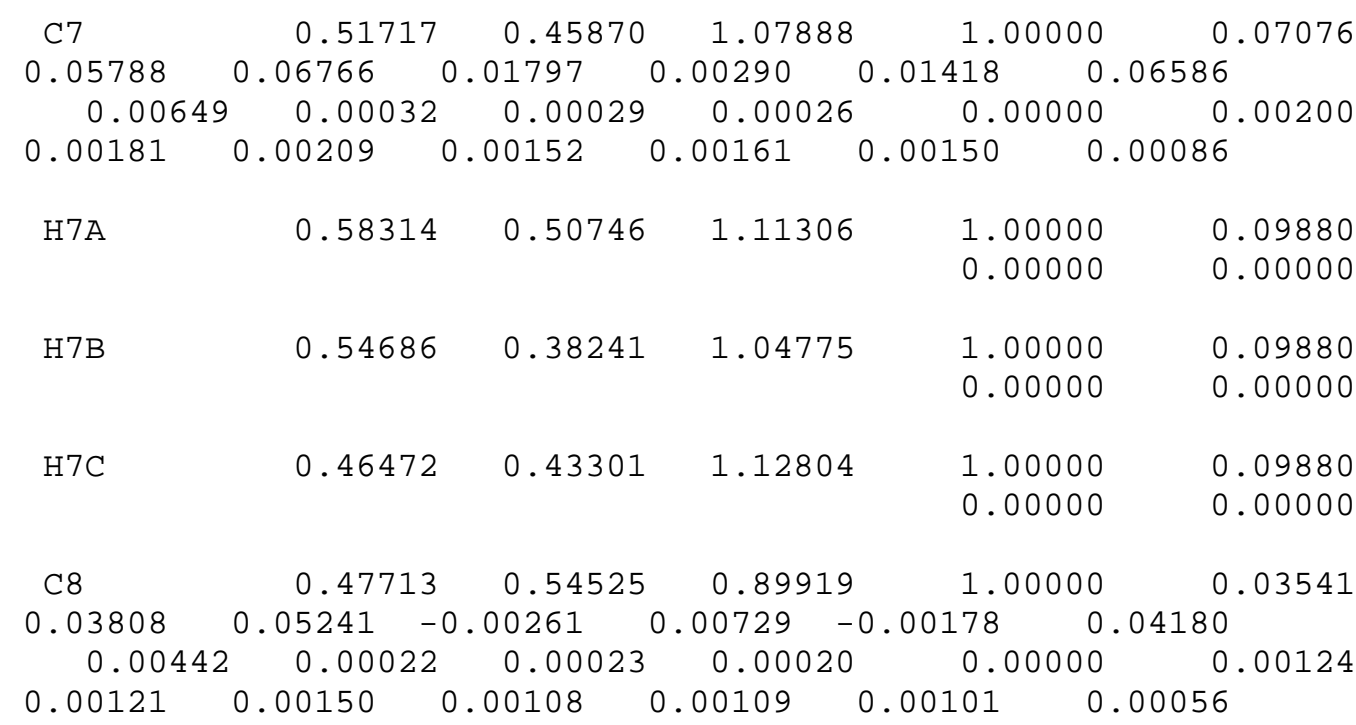
04
$\begin{array}{lll}0.55279 & 0.48033 \quad 0.86575\end{array}$
1.00000
$\odot .05196$
$\begin{array}{llllll}0.05012 & 0.07508 & -0.00178 & 0.02233 & 0.01510 & 0.05787\end{array}$
0.00388
0.00017
$0.00019 \quad 0.00017$
0.00000
0.00112
$\begin{array}{llllll}0.00103 & 0.00130 & 0.00096 & 0.00100 & 0.00089 & 0.00052\end{array}$

$\begin{array}{ccccccc}\text { C9 } & 0.39749 & 0.64605 & 0.84361 & 1.00000 & 0.03273 \\ 0.04451 & 0.03486 & -0.00525 & 0.00591 & -0.000039 & 0.03722 \\ 0.00422 & 0.00020 & 0.00023 & 0.00018 & 0.00000 & 0.00118 \\ 0.00125 & 0.00124 & 0.00096 & 0.00095 & 0.00098 & 0.00052\end{array}$

$\begin{array}{llllll}0.04528 & 0.03758 & 0.00207 & 0.00836 & 0.00223 & 0.03897\end{array}$

$\begin{array}{llllll}0.00446 & 0.00022 & 0.00023 & 0.00019 & 0.00000 & 0.00122\end{array}$

$\begin{array}{llllll}0.00125 & 0.00122 & 0.00100 & 0.00097 & 0.00104 & 0.00052\end{array}$

$\begin{array}{llllll}\text { C11 } & 0.59675 & 0.77172 & 0.83405 & 1.00000 & 0.03954\end{array}$

$\begin{array}{llllll}0.03811 & 0.04824 & 0.00190 & 0.01699 & -0.00254 & 0.04102\end{array}$
0.00466
0.00022
$0.00023 \quad 0.00020$
0.00000
0.00132

$\begin{array}{llllll}0.00115 & 0.00141 & 0.00109 & 0.00111 & 0.00103 & 0.00056\end{array}$

$\begin{array}{llllll}\mathrm{C} 12 & 0.64614 & 0.74725 & 0.74346 & 1.00000 & 0.05506\end{array}$

$\begin{array}{llllll}0.06198 & 0.07029 & -0.00566 & 0.03020 & -0.00219 & 0.06061\end{array}$

$\begin{array}{llllll}0.00632 & 0.00029 & 0.00030 & 0.00025 & 0.00000 & 0.00180\end{array}$

$\begin{array}{llllll}0.00173 & 0.00211 & 0.00148 & 0.00160 & 0.00144 & 0.00082\end{array}$

$\begin{array}{lrrrrr}\text { H12 } & 0.59628 & 0.73848 & 0.68185 & 1.00000 & 0.07274 \\ & & & & 0.00000 & 0.00000 \\ \text { C13 } & 0.76728 & 0.73595 & 0.74415 & 1.00000 & 0.06324\end{array}$

$\begin{array}{lllllll}0.08279 & 0.11387 & -0.01418 & 0.05101 & 0.00037 & 0.08337\end{array}$

$\begin{array}{llllll}0.00911 & 0.00038 & 0.00038 & 0.00041 & 0.00000 & 0.00245\end{array}$

$\begin{array}{llllll}0.00246 & 0.00328 & 0.00226 & 0.00250 & 0.00191 & 0.00121\end{array}$

$\begin{array}{llllll}\text { H13 } & 0.79879 & 0.71868 & 0.68299 & 1.00000 & 0.10005 \\ & & & & 0.00000 & 0.00000 \\ \text { C14 } & 0.84177 & 0.74945 & 0.83208 & 1.00000 & 0.03785\end{array}$

$\begin{array}{llllll}0.06383 & 0.15468 & 0.00631 & 0.03463 & 0.00528 & 0.08359\end{array}$

$\begin{array}{llllll}0.00944 & 0.00034 & 0.00035 & 0.00044 & 0.00000 & 0.00161\end{array}$

$\begin{array}{llllll}0.00204 & 0.00446 & 0.00226 & 0.00231 & 0.00149 & 0.00133\end{array}$

$\begin{array}{llllll}\mathrm{H} 14 & 0.92402 & 0.74104 & 0.83152 & 1.00000 & 0.10031\end{array}$ 


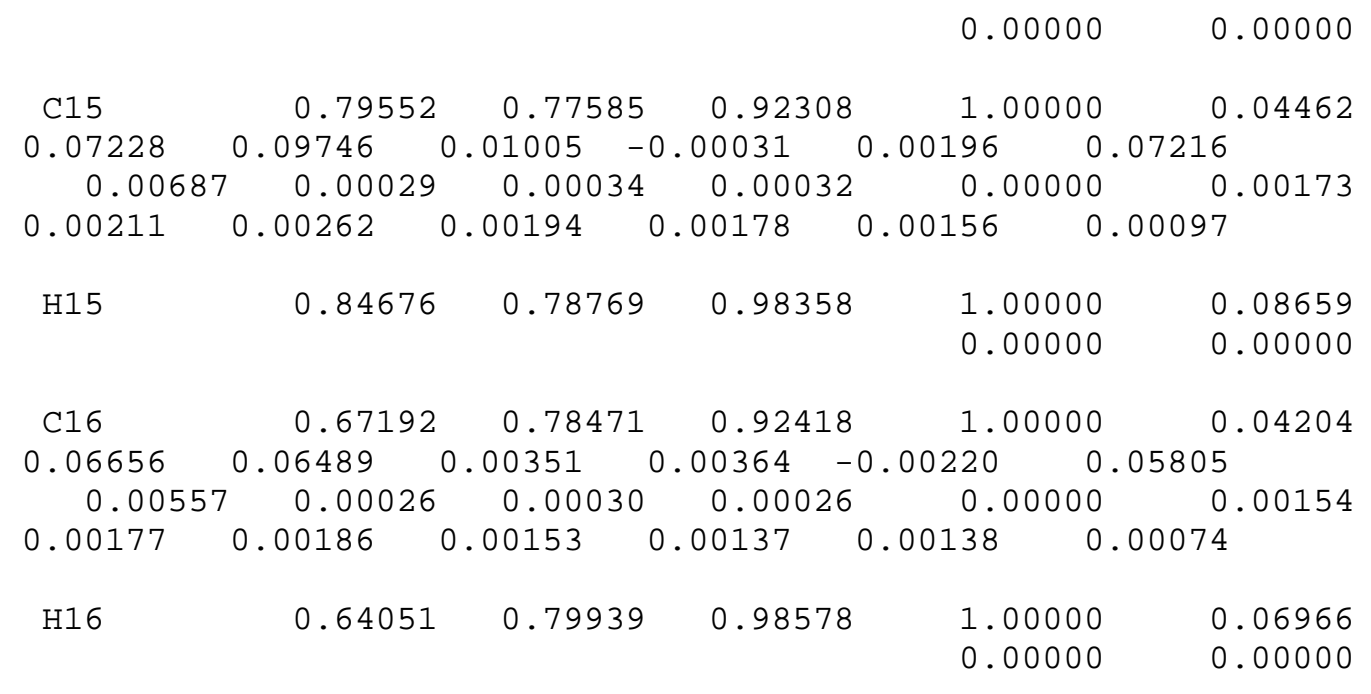

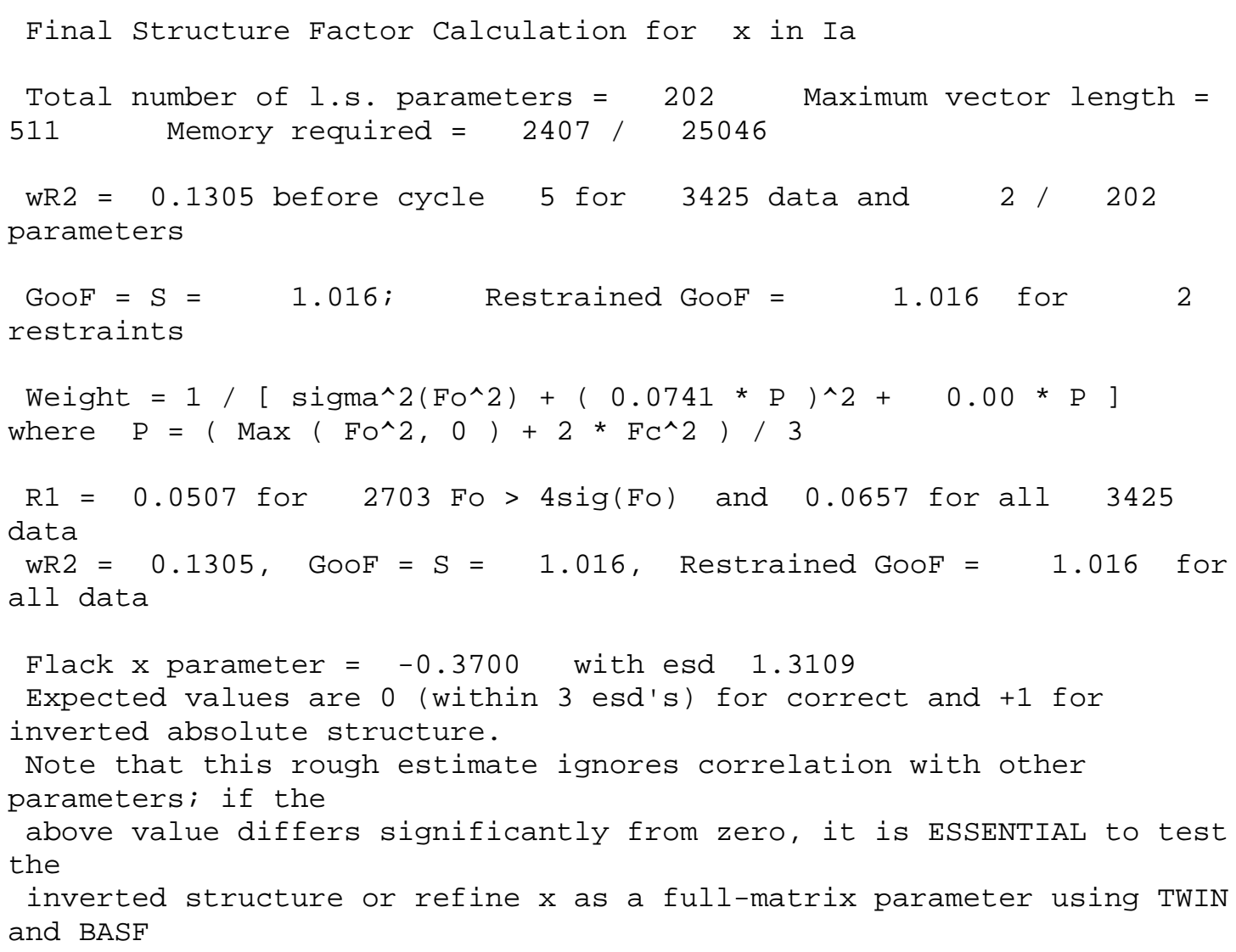

Absolute structure cannot be determined reliably

Occupancy sum of asymmetric unit $=22.00$ for non-hydrogen and 17.00 for hydrogen atoms

Principal mean square atomic displacements $U$

$\begin{array}{llll}0.0508 & 0.0337 & 0.0308 & \mathrm{~N} 1 \\ 0.0414 & 0.0373 & 0.0301 & \mathrm{C} 1 \\ 0.0438 & 0.0408 & 0.0326 & \mathrm{C} 2 \\ 0.1029 & 0.0452 & 0.0339 & 01 \\ 0.0703 & 0.0438 & 0.0311 & 02 \\ 0.0737 & 0.0594 & 0.0317 & \mathrm{C} 3\end{array}$




$\begin{array}{llll}0.0648 & 0.0387 & 0.0367 & \mathrm{C} 4 \\ 0.0437 & 0.0321 & 0.0304 & \mathrm{C} 5 \\ 0.0463 & 0.0366 & 0.0357 & \mathrm{C} 6 \\ 0.0806 & 0.0616 & 0.0333 & 03 \\ 0.0501 & 0.0435 & 0.0343 & \mathrm{~N} 2 \\ 0.0830 & 0.0755 & 0.0391 & \mathrm{C} 7 \\ 0.0530 & 0.0381 & 0.0343 & \mathrm{C} 8 \\ 0.0814 & 0.0612 & 0.0309 & 04 \\ 0.0468 & 0.0341 & 0.0307 & \mathrm{C} 9 \\ 0.0465 & 0.0389 & 0.0315 & \mathrm{C} 10 \\ 0.0552 & 0.0393 & 0.0286 & \mathrm{C} 11 \\ 0.0856 & 0.0608 & 0.0355 & \mathrm{C} 12 \\ 0.1347 & 0.0814 & 0.0340 & \mathrm{C} 13 \\ 0.1573 & 0.0637 & 0.0297 & \mathrm{C} 14 \\ 0.1032 & 0.0696 & 0.0437 & \mathrm{C} 15 \\ 0.0701 & 0.0622 & 0.0418 & \mathrm{C} 16\end{array}$

Analysis of variance for reflections employed in refinement $\mathrm{K}=$ Mean[Fo^2] / Mean[Fc^2] for group

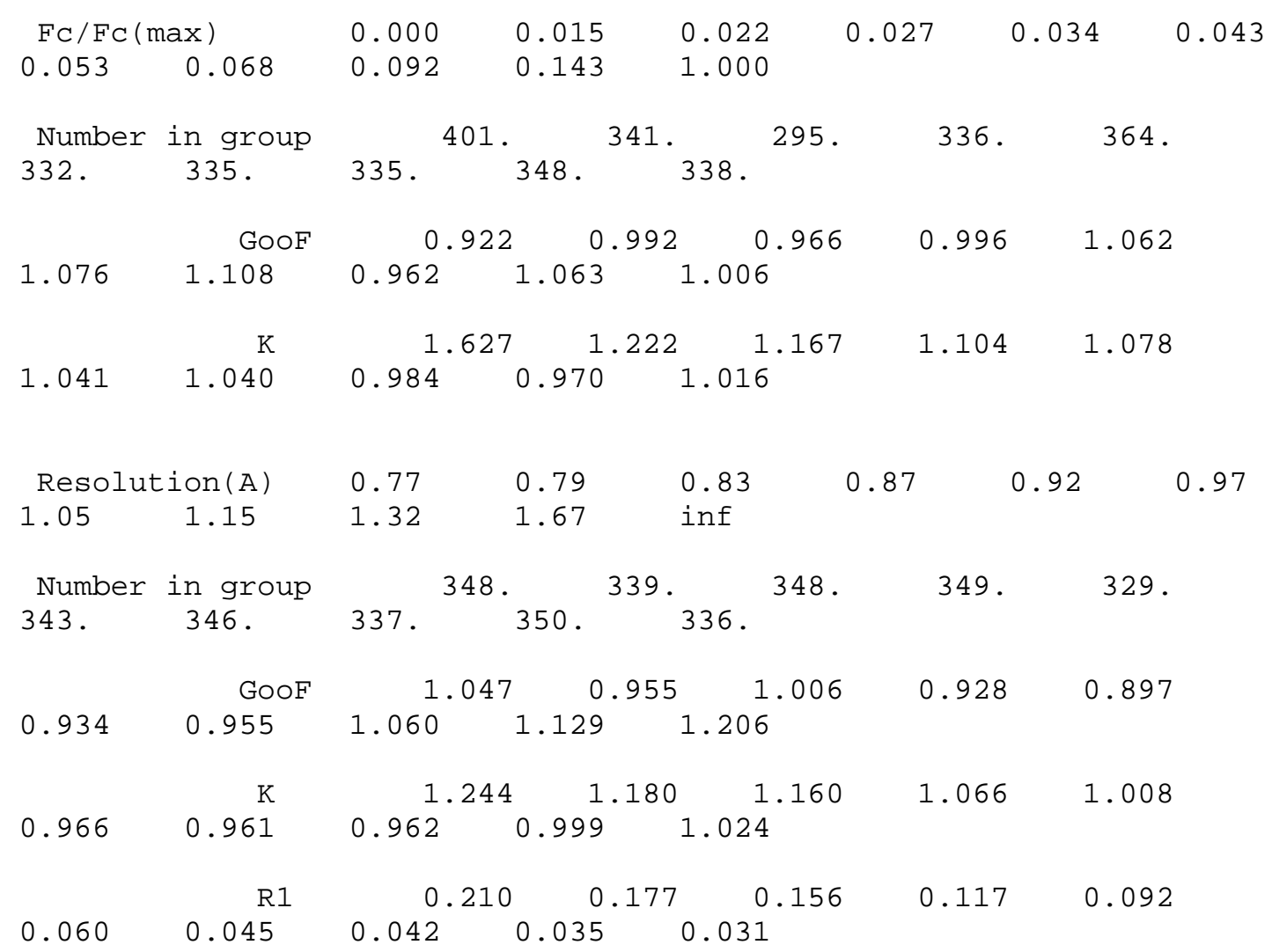

\footnotetext{
Recommended weighting scheme: WGHT $0.0726 \quad 0.0000$ Note that in most cases convergence will be faster if fixed weights (e.g. the default WGHT $\odot .1$ ) are retained until the refinement is virtually complete, and only then should the above recommended values be used.
}

Most Disagreeable Reflections (* if suppressed or used for Rfree)

$$
\mathrm{h} k 1 \quad \mathrm{Fo}^{\wedge} 2 \quad \mathrm{Fc}^{\wedge} 2 \operatorname{Delta}\left(\mathrm{F}^{\wedge} 2\right) / \mathrm{esd} F \mathrm{Fc} F \mathrm{Fax}(\max
$$


Resolution(A)

\begin{tabular}{|c|c|c|c|c|c|c|}
\hline $183^{1}$ & 4 & -5 & 140.85 & 103.36 & 3.91 & 0.057 \\
\hline $1.63^{-2}$ & 6 & 0 & 96.32 & 63.52 & 3.85 & $\odot .045$ \\
\hline $4.51^{2}$ & $\odot$ & -2 & 1463.23 & 1948.26 & 3.65 & $\odot .248$ \\
\hline $1.23^{-2}$ & 8 & 2 & 32.90 & 55.53 & 3.58 & $\odot .042$ \\
\hline $1.75^{-1}$ & 2 & 7 & 1821.15 & 1403.04 & 3.41 & $\odot .210$ \\
\hline $1.19^{9}$ & 2 & 1 & 86.57 & 57.15 & 3.41 & $\odot .042$ \\
\hline $1.99^{\circ}$ & 2 & -6 & 181.61 & 138.88 & 3.41 & 0.066 \\
\hline $1.71^{\circ}$ & 6 & 0 & 62.74 & 84.98 & 3.39 & $\odot .052$ \\
\hline $0.79^{14}$ & 2 & -2 & 43.78 & 13.39 & 3.38 & 0.021 \\
\hline $1.45^{-4}$ & 1 & -7 & 182.44 & 129.09 & 3.36 & 0.064 \\
\hline $1.99^{-5}$ & 2 & -1 & 213.46 & 164.76 & 3.26 & $\odot .072$ \\
\hline $1.26^{\circ}$ & 8 & 2 & 64.42 & 93.19 & 3.17 & $\odot .054$ \\
\hline $1.92^{-2}$ & 5 & 1 & 321.03 & 414.92 & 3.16 & 0.114 \\
\hline $1.51^{-4}$ & 5 & -3 & 201.50 & 263.41 & 3.16 & $\odot .091$ \\
\hline $1.53^{5}$ & 2 & 5 & 23.16 & 11.59 & 3.13 & 0.019 \\
\hline $1.45^{4}$ & 1 & 7 & 177.79 & 129.45 & 3.10 & $\odot .064$ \\
\hline$\odot .91^{-7}$ & 8 & -5 & 15.69 & 37.54 & 2.99 & 0.034 \\
\hline $1.10^{10}$ & $\odot$ & -4 & 302.40 & 402.26 & 2.98 & 0.113 \\
\hline $0.83^{3}$ & 12 & 1 & 41.23 & 14.43 & 2.98 & 0.021 \\
\hline $1.05^{5}$ & 4 & 9 & 49.51 & 25.28 & 2.96 & $\odot .028$ \\
\hline $0.83^{-10}$ & 5 & -7 & 48.00 & 17.77 & 2.95 & $\odot .024$ \\
\hline $1.35^{2}$ & 1 & 9 & 238.32 & 308.04 & 2.87 & $\odot .099$ \\
\hline $1.28^{-4}$ & 5 & 7 & 100.48 & 66.90 & 2.84 & $\odot .046$ \\
\hline$\odot .84^{11}$ & 7 & -2 & 127.95 & 84.74 & 2.80 & 0.052 \\
\hline $1.00^{-3}$ & 8 & -7 & 54.50 & 28.51 & 2.78 & $\odot .030$ \\
\hline $1.08^{-1}$ & 5 & -10 & 342.98 & 437.92 & 2.74 & 0.118 \\
\hline$\odot .82^{5}$ & 5 & -14 & 57.20 & 30.04 & 2.74 & 0.031 \\
\hline $2.00^{-2}$ & 3 & 5 & 169.18 & 124.74 & 2.73 & $\odot .063$ \\
\hline $1.39^{6}$ & 1 & -7 & 41.42 & 23.61 & 2.73 & 0.027 \\
\hline $1.53^{-5}$ & 2 & -5 & 25.16 & 11.56 & 2.69 & ๑. 019 \\
\hline $1.22^{-3}$ & 6 & 7 & 2.24 & 11.75 & 2.69 & 0.019 \\
\hline
\end{tabular}




\begin{tabular}{|c|c|c|c|c|c|c|}
\hline $07^{-2}$ & 9 & 7 & 7.39 & 21.63 & 2.69 & 0.026 \\
\hline $\begin{array}{l}0.97 \\
2.22\end{array}$ & 2 & 2 & 963.56 & 792.43 & 2.67 & 0.158 \\
\hline $\begin{array}{l}7.27 \\
1.27\end{array}$ & 3 & -6 & 562.52 & 696.40 & 2.63 & 0.148 \\
\hline $1.78^{1}$ & 4 & 5 & 1214.69 & 999.10 & 2.61 & 0.177 \\
\hline $1.64^{6}$ & 3 & -1 & 296.12 & 241.62 & 2.60 & 0.087 \\
\hline $0.84^{10}$ & 8 & $\odot$ & 84.33 & 53.43 & 2.60 & 0.041 \\
\hline $1.16^{2}$ & 8 & 4 & 107.86 & 155.29 & 2.59 & 0.070 \\
\hline $1.64^{-6}$ & 3 & 1 & 295.90 & 241.01 & 2.58 & $\odot .087$ \\
\hline $0.78^{13}$ & 5 & 2 & 35.95 & 2.38 & 2.58 & 0.009 \\
\hline $1.40^{4}$ & 6 & 2 & 55.83 & 77.23 & 2.57 & 0.049 \\
\hline $1.02^{1}$ & 10 & 1 & -1.58 & 7.13 & 2.51 & $\odot .015$ \\
\hline $1.03^{-2}$ & 9 & 5 & 32.58 & 17.04 & 2.51 & 0.023 \\
\hline $1.47^{1}$ & 3 & -8 & 645.80 & 537.71 & 2.49 & 0.130 \\
\hline $1.35^{-4}$ & 5 & -5 & 115.19 & 89.79 & 2.48 & 0.053 \\
\hline $1.16^{-6}$ & $\odot$ & -8 & 29.85 & 54.02 & 2.47 & $\odot .041$ \\
\hline $0.79^{\odot}$ & 12 & -6 & 21.54 & 42.03 & 2.46 & $\odot .036$ \\
\hline $1.20^{-2}$ & 2 & -10 & 31.49 & 18.19 & 2.45 & $\odot .024$ \\
\hline $1.99^{5}$ & 2 & 1 & 200.83 & 165.10 & 2.45 & 0.072 \\
\hline $1.78^{-1}$ & 4 & -5 & 1195.32 & 999.25 & 2.45 & 0.177 \\
\hline
\end{tabular}

Bond lengths and angles

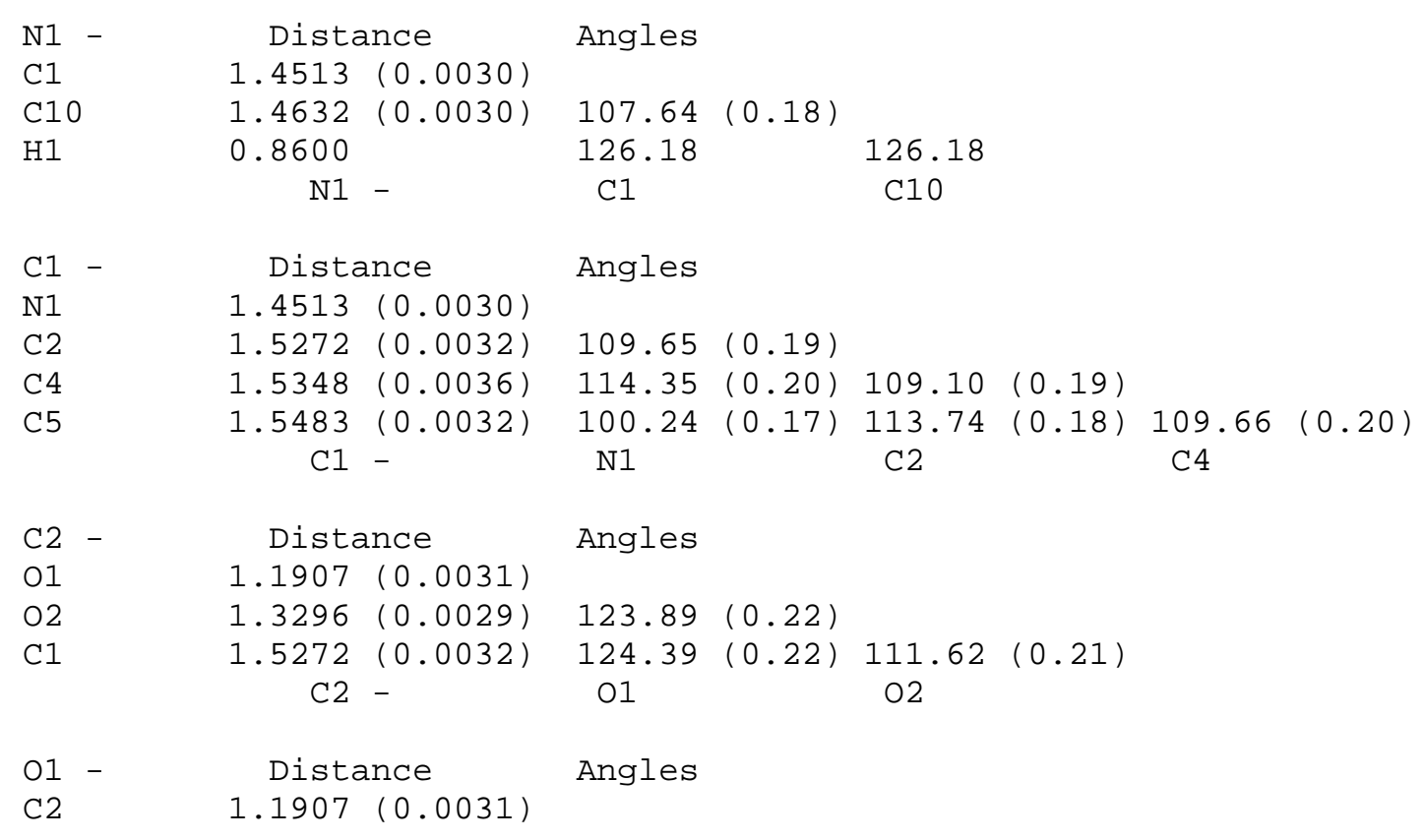


$01-$

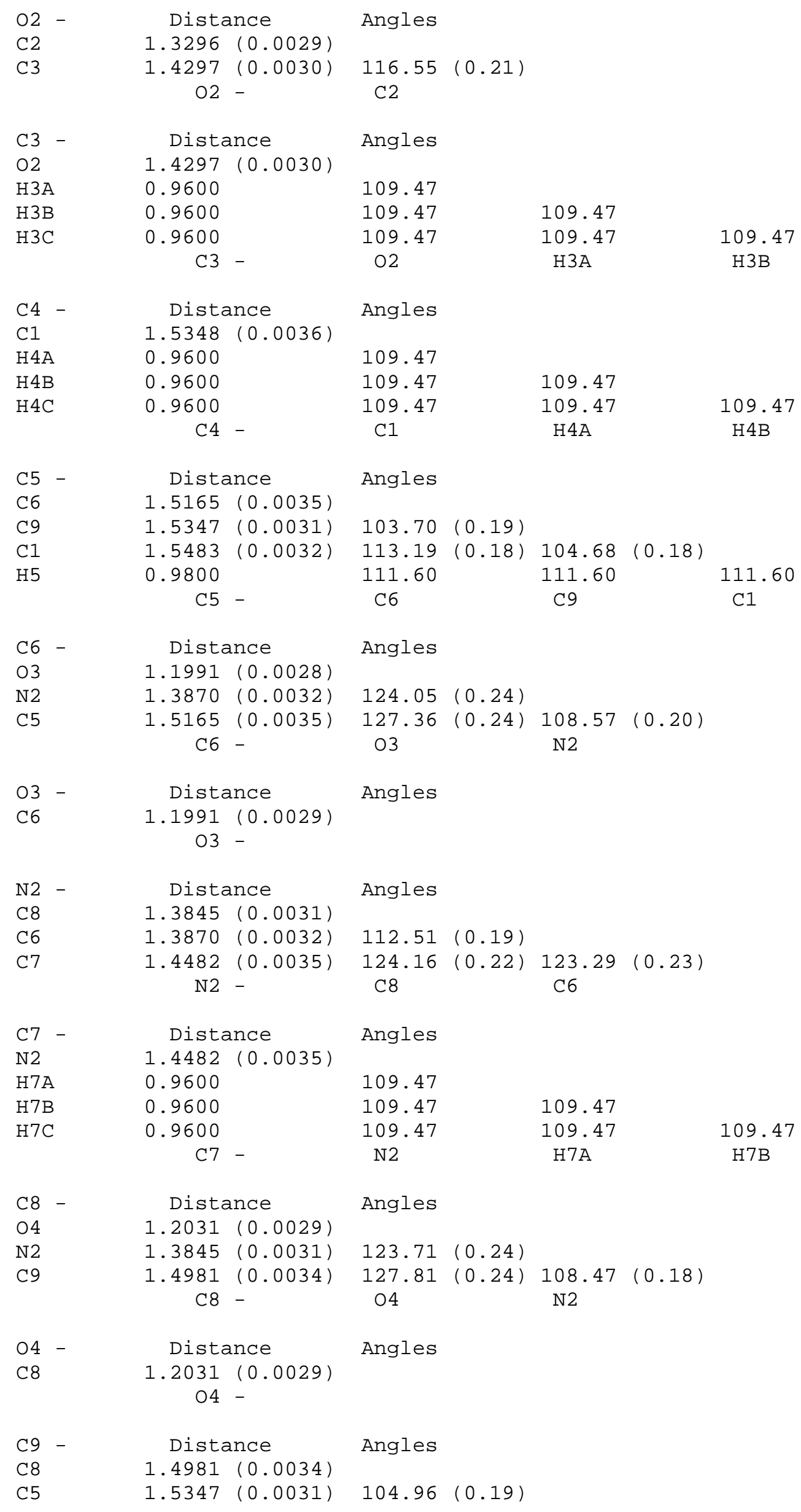




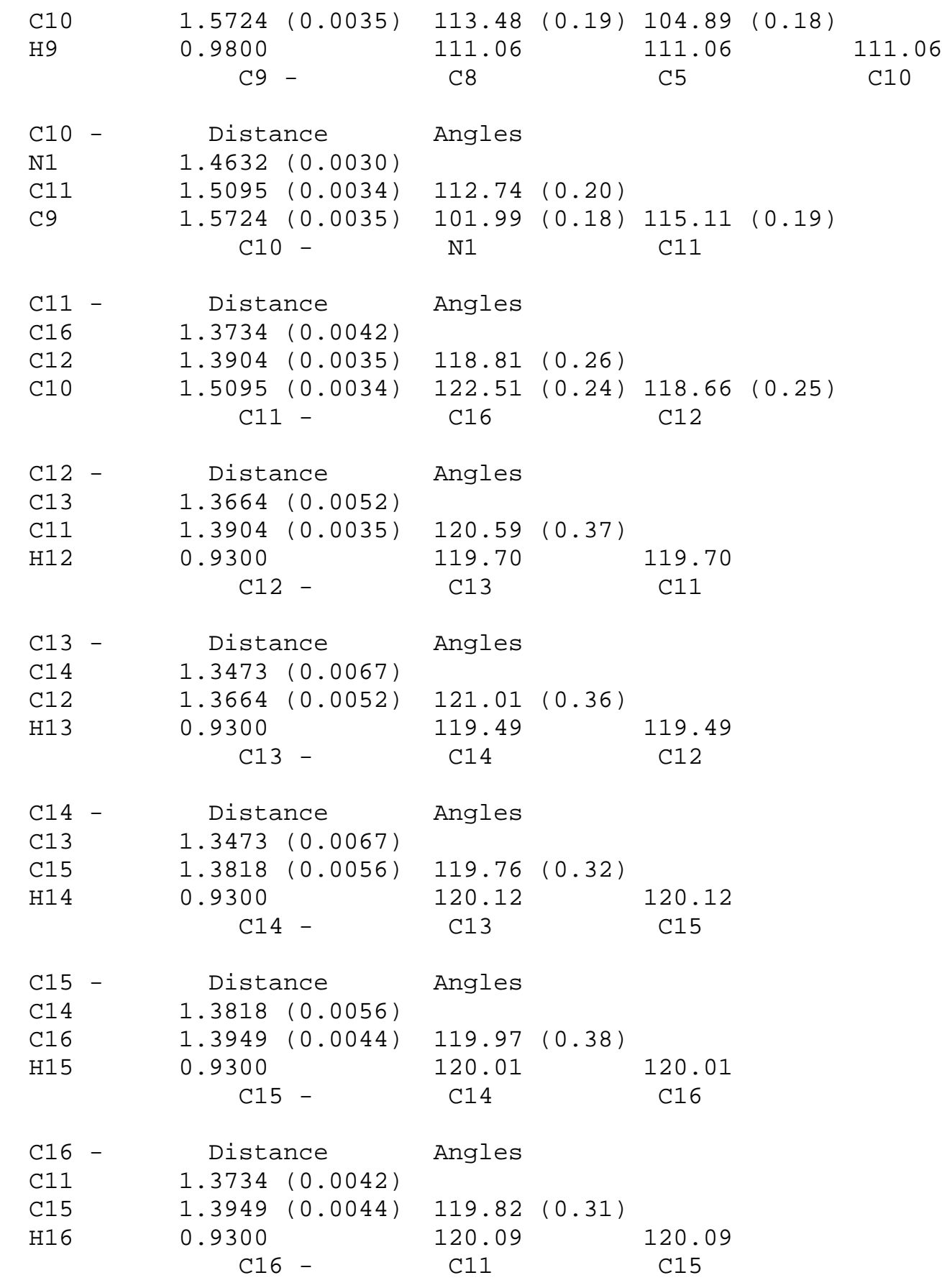

FMAP and GRID set by program

FMAP $2 \quad 2 \quad 15$

GRID $\quad-2.083 \quad-2 \quad-2 \quad 2.083 \quad 2 \quad 2$

$\mathrm{R} 1=0.0571$ for 1746 unique reflections after merging for Fourier

Electron density synthesis with coefficients Fo-Fc

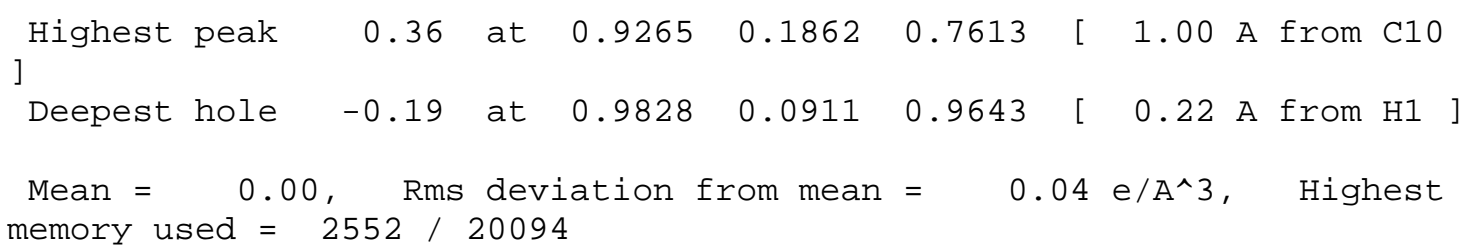


Fourier peaks appended to .res file

$\begin{array}{lllllll}x & y & z & \text { sof } & U & \text { Peak Distances }\end{array}$ to nearest atoms (including symmetry equivalents)

\begin{tabular}{|c|c|c|c|c|c|c|c|c|c|}
\hline Q1 & 1 & 0.4265 & 0.8138 & 0.7613 & 1.00000 & 0.05 & 0.36 & 1.00 & C10 \\
\hline 2.05 & N1 & $2.08 c 9$ & $2.08 \quad$ C11 & & & & & & \\
\hline Q2 & 1 & 0.4282 & 0.9445 & ๑. 8982 & 1.00000 & 0.05 & 0.27 & 0.86 & $\mathrm{H} 1$ \\
\hline 0.92 & N1 & $1.93 \mathrm{C} 1$ & 1.97 C10 & & & & & & \\
\hline Q3 & 1 & 0.5181 & 0.7742 & 0.8267 & $1.0000 \odot$ & 0.05 & 0.15 & 0.64 & C10 \\
\hline 0.88 & C11 & 1.85 & 1.9 & & & & & & \\
\hline Q4 & 1 & 0.3350 & 0.5816 & 1.1610 & $1.0000 \odot$ & 0.05 & 0.15 & 0.93 & 03 \\
\hline 1.95 & $\mathrm{C} 6$ & $2.09 \mathrm{H} 4 \mathrm{C}$ & $9 \mathrm{H} 7 \mathrm{C}$ & & & & & & \\
\hline Q5 & 1 & 0.2613 & 0.8 & 0.8739 & $1.000 \odot \odot$ & 0.05 & 0.15 & $\odot .76$ & C4 \\
\hline 0.80 & C1 & $1.32 \mathrm{H} 4 \mathrm{~A}$ & 1.4 & & & & & & \\
\hline Q6 & 1 & 0.4395 & 0.7226 & 0.8397 & $1.0 \odot \odot \odot \odot$ & 0.05 & 0.14 & 0.66 & C10 \\
\hline 0.92 & C9 & $1.60 \mathrm{H} 9$ & $1.71 \mathrm{~N} 1$ & & & & & & \\
\hline Q7 & 1 & 0.2039 & 0.8244 & 1.0396 & 1.00000 & 0.05 & 0.14 & 0.73 & 02 \\
\hline 0.93 & C2 & $1.84 \mathrm{C3}$ & 1.86 & & & & & & \\
\hline Q8 & 1 & 0.6077 & 0.7840 & 0.7770 & $1 . \odot \odot \odot \odot \odot$ & 0.05 & 0.13 & 0.75 & C12 \\
\hline & & & & & & & & & \\
\hline
\end{tabular}

Shortest distances between peaks (including symmetry equivalents)

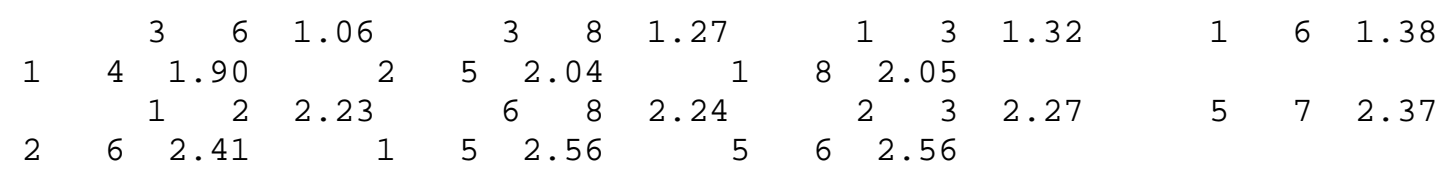

Time profile in seconds

$\odot .03:$ Read and process instructions

0.00 : Fit rigid groups

$\odot .00$ : Interpret restraints etc.

$\odot .00$ : Generate connectivity array

$\odot . \odot \odot:$ Analyse DFIX/DANG restraints

0.00 : Analyse SAME/SADI restraints

$\odot .00$ : Generate CHIV restraints

0.00 : Check if bonds in residues restrained

0.00 : Generate DELU restraints

0.00 : Generate SIMU restraints

0.00 : Generate ISOR restraints

0.00 : Generate NCSY restraints

0.00 : Analyse other restraints etc.

0.13 : Read intensity data, sort/merge etc.

0.00 : Set up constraints

๑.००: OSF, H-atoms from difference map

0.00 : Set up l.s. refinement

0.00 : Generate idealized $\mathrm{H}$-atoms

1.00: Structure factors and derivatives

0.97 : Sum 1.s. matrices

$\odot .0 \odot$ : Generate and apply antibumping restraints

$\odot .00:$ Apply other restraints

0.06 : Solve l.s. equations

$\odot . \odot \odot:$ Generate HTAB table

0.02 : Other dependent quantities, CIF, tables

0.06 : Analysis of variance

0.00 : Merge reflections for Fourier and .fcf

0.02 : Fourier summations

0.08 : Peaksearch

$\odot . \odot \odot:$ Analyse peaklist 


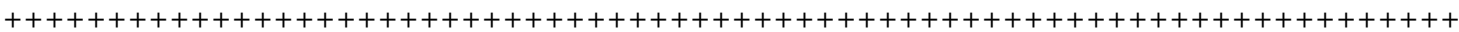
$++++++$

$+\mathrm{X}$

finished at 09:54:54 Total CPU time:

2.4

$\operatorname{secs}+$

++++++++++++++++++++++++++++++++++++++++++++++++++++++++++++++++++++++ $++++++$ 